This is the submitted version of the article:

Belec B., Dražic G., Gyergyek S., Podmiljšak B., Goršak T., Komelj M., Nogués J., Makovec D.. Novel Ba-hexaferrite structural variations stabilized on the nanoscale as building blocks for epitaxial bi-magnetic hard/soft sandwiched maghemite/hexaferrite/maghemite nanoplatelets with out-of-plane easy axis and enhanced magnetization. Nanoscale, (2017). 9. : 17551 - . 10.1039/c7nr05894b.

Available at: https://dx.doi.org/10.1039/c7nr05894b 


\title{
Novel Ba-hexaferrite structural variations stabilized on the nanoscale as building blocks for epitaxial bi-magnetic hard/soft sandwiched maghemite/hexaferrite/maghemite nanoplatelets with out-of-plane easy axis and enhanced magnetization
}

\begin{abstract}
B. Belec, ${ }^{a, b}$ G. Dražić, c S. Gyergyek, ${ }^{a}$ B. Podmiljšak, ${ }^{d}$ T. Goršak, ${ }^{a, b}$ M. Komelj, ${ }^{d}$ J. Nogués, e,f and D. Makovec ${ }^{a, b^{*}}$

Atomic-resolution scanning-transmission electron microscopy showed that barium hexaferrite (BHF) nanoplatelets display a distinct structure, which represents a novel structural variation of hexaferrites stabilized on the nanoscale. The structure can be presented in terms of two alternating structural blocks stacked across the nanoplatelet: a hexagonal $\left(\mathrm{BaFe}_{6} \mathrm{O}_{11}\right)^{2-} \mathrm{R}$ block and a cubic $\left(\mathrm{Fe}_{6} \mathrm{O}_{8}\right)^{2+}$ spinel $\mathrm{S}$ block. The structure of the BHF nanoplatelets comprises only two, or rarely three, $\mathrm{R}$ blocks and always terminates at the basal surfaces with the full $S$ blocks. The structure of a vast majority of the nanoplatelets can be described with a $\mathrm{SR}^{*} \mathrm{~S}^{*} \mathrm{RS}$ stacking order, corresponding to a $\mathrm{BaFe}_{15} \mathrm{O}_{23}$ composition. The nanoplatelets display a large, uniaxial magnetic anisotropy with the easy axis perpendicular to the platelet, which is a crucial property enabling different novel applications based on aligning the nanoplatelets with applied magnetic fields. However, the HF nanoplatelets exhibit a modest saturation magnetization, $\boldsymbol{M}_{\mathbf{s}}$, of just over 30 emu/g. Given the cubic $\mathrm{S}$ block termination of the platelets, layers of maghemite, $\gamma-\mathrm{Fe}_{2} \mathrm{O}_{3},(\mathrm{M})$, with a cubic spinel structure, can be easily grown epitaxially on the surfaces of the platelets, forming a sandwiched M/BHF/M platelet structure. The exchange-coupled composite nanoplatelets exhibit a remarkably uniform structure, with an enhanced $\boldsymbol{M}_{\mathbf{s}}$ of more than 50 emu/g while essentially maintaining the out-of-plane easy axis. The enhanced $\boldsymbol{M}_{\mathbf{s}}$ could pave the way for their use in diverse plateletbased magnetic applications.
\end{abstract}

\section{Introduction}

Single-domain magnetic nanoparticles with an anisotropic shape have shown great potential in innovative applications related to their ability to be effectively aligned by an applied magnetic field. When the anisotropic magnetic particle is subjected to a magnetic field $\mathrm{H}$ the direction of its magnetic moment $\mathrm{m}$ will align with the field, resulting in a driving torque $\tau_{\boldsymbol{m}}=\boldsymbol{m} \times \boldsymbol{H}$ that can be transferred to the surroundings. This effect is interesting for new magnetically responsive materials, such as magneto-optic composites, ${ }^{1}$ soft magneto-electrics, ${ }^{2-7}$ and magneto-viscous fluids. ${ }^{4,8}$ The magneto-mechanical actuation of nanoparticles in a low-frequency alternating magnetic field can be used for diverse biomedical applications, such as the remote control of nanomedicines, drug-delivery systems and cell apostosis. ${ }^{9-11}$ Moreover, the effective coupling of the nanoparticle magnetic moments with a magnetic field can be used in sensors. ${ }^{12-15}$

In particular, magnetic nanoplatelets that display a high uniaxial magnetocrystalline anisotropy with an easy axis perpendicular to the platelet can be especially appropriate for certain applications. Barium hexaferrite $\left(\mathrm{BaFe}_{12} \mathrm{O}_{19}, \mathrm{BHF}\right)$ nanoplatelets are a classic example of this type of magnetic structure. ${ }^{1,16}$ The BHF crystalizes in a magnetoplumbite structure that can be represented as a hexagonal close-packed stacking of oxygen and barium ions with smaller iron ions positioned at the interstitial sites. The structure can be subdivided into two alternating structural blocks stacked along the c-direction: a hexagonal " $\mathrm{R}$ " block $\left(\left(\mathrm{BaFe}_{6} \mathrm{O}_{11}\right)^{2-}\right)$ and a cubic "S" block $\left(\left(\mathrm{Fe}_{6} \mathrm{O}_{8}\right)^{2+}\right)^{17,18}$ (Fig. $\left.1(\mathrm{~d})\right)$. The unit cell (S.G.: $\mathrm{P} 63 / \mathrm{mmc}, \mathrm{a}=0.588 \mathrm{~nm}, \mathrm{c}=2.318 \mathrm{~nm}$ ) can be illustrated by the RSR*S* stacking sequence, where the asterisk denotes the rotation of the block by $180^{\circ}$ around the hexagonal c-axis. Interestingly, the growth of the BHF crystals is limited in the cdirection, resulting in BHF nanoparticles growing in the form of thin hexagonal platelets. ${ }^{16}$ Due to its anisotropic crystal structure BHF displays a uniaxial magnetocrystalline anisotropy with the easy axis aligned parallel to the crystallographic caxis, and consequently perpendicular to the nanoplatelet surafce. ${ }^{17}$ Importantly, due to its high magnetocrystalline anisotropy $\left(\mathrm{K}_{\mathrm{i}}=3.3 \times 10^{4} \mathrm{~J} / \mathrm{m}^{3} \text { in the bulk }\right)^{17}$ the $\mathrm{BHF}$ nanoplatelets will tend to rotate so as to align with an external magnetic field than reversing the magnetic moment. The effect of a magnetic field on the nanoplatelet, e.g., a driving torque, is proportional to the nanoplatelet's magnetic moment $\boldsymbol{m}$ (i.e., the product of its volume $\boldsymbol{V}$ and saturation magnetization $\boldsymbol{M}_{\mathbf{s}}, \boldsymbol{m}=\boldsymbol{V} . \boldsymbol{M}_{\mathbf{s}}$ ). Since for many applications the nanoparticle size is usually required to remain small, the $M_{\mathbf{s}}$ should be increased to maximize the effect of the field on the nanoplatelet. In bulk form BHF displays a moderate $\boldsymbol{M}_{\mathbf{s}}$ of 72 emu/g, ${ }^{17}$ but in nanoplatelet form the $\boldsymbol{M}_{\mathbf{s}}$ is usually significantly smaller. ${ }^{16,19-21}$ For example, BHF nanoplatelets can be synthesized using hydrothermal methods; ${ }^{16,19-21}$ however, due to the poor structural order related to the small thickness of the nanoplatelets, which is commensurate with the unit cell dimension in the c-direction, they usually display a relatively low $M_{s}$, not exceeding $20 \mathrm{emu} / \mathrm{g} .{ }^{16}$ In fact, due to their size, comparable with the lattice parameter in the c-direction, it is expected that BHF nanoplatelets will adopt a certain equilibrium structure that is different from bulk. This structure will also define the composition of the nanoplatelets, which can differ from the stoichiometric $\mathrm{BaFe}_{12} \mathrm{O}_{19}$ composition. For example, if the structure were to terminate with layers containing mainly $\mathrm{Ba}$ atoms, the nanoplatelet would be Barich. Note that although the structure of ultrafine hexaferrite 
nanoplatelets has been studied using different methods, including X-ray diffractometry (XRD), high-resolution transmission electron microscopy (HRTEM), X-ray absorption fine structure (XAFS), and Mössbauer spectroscopy, the exact structure remains to be elucidated. ${ }^{16}$

In this work we determine, for the first time, the equilibrium structure and the composition of thin BHF nanoplatelets by using atomic-resolution high-angle annular dark-field (HAADF) imaging with a probe spherical-aberration $\left(C_{S}\right)$ corrected scanning-transmission electron microscope (STEM). The structures and the compositions of the nanoplatelets differ from the bulk and can therefore be viewed as novel structural variations of hexaferrites stabilized on the nanoscale.

To improve the potential of BHF nanoplatelets for applications, an increase in $\boldsymbol{M}_{\mathbf{s}}$ is essential. For example, doping BHF with Sc leads to significant improvement in the $\boldsymbol{M}_{\mathbf{s}}$ to above $30 \mathrm{emu} / \mathrm{g}$, while preserving the out-of-plane easy axis. ${ }^{15}$ This has led to the use of $\mathrm{BFH}$ in diverse applications such as ferromagnetic liquid crystals $^{3,22}$ and magneto-optical composites. ${ }^{1}$ These applications of hard-magnetic nanoplatelets are very encouraging; however, they also revealed that a further improvement in their $\boldsymbol{M}_{\mathbf{S}}$ is necessary. An alternative approach to enhance $M_{s}$ in hard-magnetic materials is the exchange coupling low- $\boldsymbol{M}_{\mathbf{s}}$ materials (e.g., BHF) with high-Ms softmagnetic materials. ${ }^{23-36}$ Actually, in recent years, there has been an increased interest in hard-soft-magnetic core/shell nanoparticles due to the large number of potential applications. $^{25}$ However, most of the studied systems have polycrystalline shells, which inevitably results in sub-optimal properties. ${ }^{25}$ Although some epitaxial, bi-magnetic, core/shell nanoparticles can be found in the literature, ${ }^{26-36}$ they often rely on different oxidation states of the same material ${ }^{26-30}$ or different compositions of the same structure. ${ }^{31-33}$ In contrast, reports of epitaxial, heterostructured magnetic core/shell materials are rather scarce. ${ }^{34-36}$

In the present work the $\boldsymbol{M}_{\mathbf{s}}$ of hard-magnetic Sc-doped barium hexaferrite nanoplatelets was increased by coupling them with high- $\boldsymbol{M}_{\mathbf{s}}$ soft-magnetic epitaxial layers of maghemite iron oxide $\left(\gamma-\mathrm{Fe}_{2} \mathrm{O}_{3}\right)$ grown on their basal surfaces. The "sandwiched" nanoplatelets composed of a hexaferrite platelet core in between two epitaxial maghemite layers show a homogeneous magnetization, indicating a strong exchange coupling, resulting in single-phase-like behaviour. Composite nanoplatelets with a homogeneous magnetization $\boldsymbol{M}_{\mathbf{s}}$ above $50 \mathrm{emu} / \mathrm{g}$ and an out-of-plane easy axis were synthesized by optimizing the thickness of the maghemite layer.

\section{Experimental}

\section{Synthesis of hexaferrite nanoplatelets.}

Sc-doped barium hexaferrite nanoplatelets (BSHF) with the nominal composition $\mathrm{BaFe}_{10.5} \mathrm{Sc}_{1.5} \mathrm{O}_{12}$ were synthesized using a hydrothermal method. ${ }^{21}$ Metal nitrates of $\mathrm{Ba}^{2+}(4.16 \mathrm{mmol})$, $\mathrm{Fe}^{3+}(18.2 \mathrm{mmol})$ and $\mathrm{Sc}^{3+}(2.6 \mathrm{mmol})$ (the used materials are listed in electronic supplementary information (ESI)) were dissolved in $200 \mathrm{~mL}$ of distilled water. The solution was rapidly mixed into $200 \mathrm{~mL}$ of aqueous $\mathrm{NaOH}(1.13 \mathrm{~mol})$ to coprecipitate the corresponding hydroxides. The slurry was then sealed in an inconel pressure vessel (Parr Instrument Company) and heated to $240{ }^{\circ} \mathrm{C}$ at a heating rate of $3{ }^{\circ} \mathrm{C} / \mathrm{min}$. Afterwards, the autoclave was allowed to cool naturally to room temperature. The product was subsequently washed, first with diluted nitric and, finally, with distilled water. Washing with nitric acid dissolves any Ba-rich phases that may form due to the excess $\mathrm{Ba}$.

\section{Deposition of maghemite onto the hexaferrite nanoplatelets.}

Maghemite was deposited onto the BSHF core nanoplatelets using the controlled co-precipitation of the $\mathrm{Fe}^{3+} / \mathrm{Fe}^{2+}$ ions in their aqueous suspension. ${ }^{37}$ First, the BSHF nanoplatelets (40 $\mathrm{mg}$ ) were dispersed in diluted nitric acid $(80 \mathrm{~mL})$ at a $\mathrm{pH}$ of 4.5 , since, due to the highly positive $\zeta$-potential (ESI, Fig. S1), at this $\mathrm{pH}$ a stable aqueous colloidal suspension is obtained. The suspension was heated to $60{ }^{\circ} \mathrm{C}$ in an argon flow to expel any oxygen. Then, $\left[\mathrm{Fe}\left(\left(\mathrm{H}_{2} \mathrm{~N}\right)_{2} \mathrm{C}=\mathrm{O}\right)_{6}\right]\left(\mathrm{NO}_{3}\right)_{3} \quad\left(\mathrm{Fe}^{3+}\right.$-urea complex, ${ }^{38}$ $0.09-0.18 \mathrm{mmol})$ and $\mathrm{FeCl}_{2}(0.045-0.9 \mathrm{mmol})$ were dissolved in the suspension and stirred for 10 minutes. During this time, at a low $\mathrm{pH}$ of 2.2, the $\mathrm{Fe}^{3+}$ ions released by the slow thermal decomposition of the $\mathrm{Fe}^{3+}$-urea complex gradually hydrolyse and precipitate as an iron oxide hydroxide $(\gamma-\mathrm{FeOOH}$, lepidocrocite), which heterogeneously nucleates exclusively at the surfaces of the core nanoplatelets. ${ }^{37}$ Subsequently, solid $\mathrm{Mg}(\mathrm{OH})_{2}(0.24-0.48 \mathrm{mmol})$ was added to the suspension. Its slow dissolution results in a gradual and homogeneous increase in the suspension's $\mathrm{pH}$ needed for the coprecipitation of the $\mathrm{Fe}^{2+}$ and the remaining $\mathrm{Fe}^{3+}$. At a $\mathrm{pH}$ of approximately 5.8 the $\mathrm{Fe}^{2+}$ ions precipitate and react with the previously formed $\gamma$-FeOOH at the nanoplatelets' surfaces to form a spinel iron-oxide; ${ }^{37}$ this is firstly magnetite, which then usually completely oxidizes to maghemite when exposed to the ambient air. ${ }^{39}$ After aging for $2 \mathrm{~h}$ at $60{ }^{\circ} \mathrm{C}$ the reaction mixture was allowed to cool naturally to room temperature. The product was magnetically separated, thoroughly washed and then dried in a vacuum. Interestingly, due to its simplicity and the use of inexpensive, non-toxic raw materials this method is ideal to scale-up the process for mass production. Note that the thickness of the maghemite shell can be controlled by either varying the concentration of the $\mathrm{Fe}$ precursors (see above) or by carrying out the coating process in two steps. The composite nanoplatelets are denoted as "CNPX.X", where "X.X" indicates the average maghemite shell thickness in nm. For the samples CNP1.1, CNP1.5 and CNP1.9, the Fe/BSHF molar ratio was 2.7, 3.6, and 5.4, respectively. For the two-step process (sample CNP2.5), the CNP1.9 composite nanoplatelets were subsequently coated with an additional layer of maghemite in the second step. The as-synthesized CNP1.9 were re-dispersed in a diluted aqueous solution of nitric acid at $\mathrm{pH} \sim 4$ 4.5. However, even though the composite nanoplatelets displayed a large, positive $\zeta$-potential at this $\mathrm{pH}$, they slowly agglomerated (probably due to enhanced magnetic dipolar interactions as a consequence of the improved $\boldsymbol{M}_{\mathbf{S}}$ in the CNP1.9). To prevent the agglomeration 
polyvinilpirolidone $(2 \mathrm{~g} / \mathrm{L})$ was added to the mixture. The second coating step was carried out using $40 \mathrm{mg}$ of CNP1.9+ $0.09 \mathrm{mmol}$ of $\mathrm{Fe}^{3+}$-urea complex $+0.045 \mathrm{mmol}$ of $\mathrm{Fe}^{2+}$.

\section{Characterization.}

The morpho-structural characterization of the samples was carried out by a combination of $x$-ray diffraction XRD (PANalytical $X$ ' Pert PRO), Raman spectroscopy (NT-MDT Integra Spectra for Materials Science), TEM (Jeol 2010F), and STEM (Jeol ARM 200CF). The magnetic properties were measured with a vibrating-sample magnetometer (VSM Lake Shore 7307). To minimize the particle aggregation and growth during the high-temperature magnetic measurements, the samples were coated with a silica shell. ${ }^{40}$ Details of the characterization methods are given in the Supporting Information.

\section{Results and discussion}

\section{Structure of hexaferrite and composite nanoplatelets.}

As can be seen in Fig. 1(a), the XRD pattern of the BSHF nanoplatelets resembles that of polycrystalline bulk Ba-Schexaferrite (obtained by sintering the nanoplatelets at 1200 ${ }^{\circ} \mathrm{C}$ ), although some differences can be easily observed. These differences can mainly be attributed to the platelet shape and the very small thickness of the particles; ${ }^{16,41,42}$ however, some features probably arise from some structural differences between the bulk and the nanoplatelets. The small thickness of the platelets and the associated broadening of the XRD peaks make a quantitative analysis of the XRD pattern rather complex. Similarly, the Raman spectrum of the BSHF nanoplatelets shows bands at roughly the same wavenumbers as the bulk Ba-Sc-hexaferrite ${ }^{43}$ (see ESI Fig. S2), although some discrepancies between both spectra (e.g., intensity ratios) can be easily observed. Once more, this implies that the platelets might have a distinctive crystalline structure. Again, the broadening of the bands due to the small size makes a more in-depth analysis complicated.

TEM (see Fig. 1 (b)) and STEM analyses showed that, indeed, the particles have a platelet shape with an average diameter of $42 \pm 12 \mathrm{~nm}$ (Fig. 1 (c)) and a thickness of approximately $3 \mathrm{~nm}$. The distinct crystalline structure of the BSHF nanoplatelets was examined with atomic-resolution imaging using probe $\mathrm{C}_{\mathrm{S}^{-}}$ corrected STEM. Figs. 1 (e) and (f) show the high-angle annular dark-field (HAADF) STEM image of a nanoplatelet oriented along the [11-20] direction of its hexagonal structure. Thus, the nanoplatelet is oriented edge-on, with its basal surfaces parallel to the electron beam. The image is characterized by a periodic pattern across the nanoplatelet, which reflects the stacking of the two alternating structural blocks. The structure always terminates at the basal surfaces of the nanoplatelets in the same way. However, as the image of the very surface of the free-standing nanoplatelets is somewhat affected by surface effects, it is speculative to draw conclusions about structure termination of the BSHF nanoplatelets from these images. Thus, we will discuss in more detail the structure of the BSHF platelets when examining the composite nanoplatelets.

The XRD patterns (Fig. 1 (a)) of the composite nanoplatelets showed broad peaks corresponding to both the hexaferrite and spinel structures. Similarly, Raman spectroscopy also shows the presence of hexaferrite ${ }^{43}$ and spinel ${ }^{39,44}$ bands. However, the Raman spectroscopy (ESI, Fig. S2) clearly showed that the phase with a spinel structure is actually maghemite, $\gamma$ $\mathrm{Fe}_{2} \mathrm{O}_{3}$ (the results of the Raman analysis are discussed further in the ESI).

The TEM analysis showed that the composite particles retain the platelet shape of the cores (Fig. 2(a)). Moreover, the HRTEM (Figs. 2(b) and (c)) and HAADF STEM imaging (Figs. $2(d)-(f))$ revealed that all the composite nanoplatelets display a sandwich-type structure, clearly visible when the nanoplatelet is oriented edge-on, with the basal surfaces parallel to the electron beam. The imaging of several hundred composite nanoplatelets confirmed that each one is composed of a hexaferrite (BSHF) core in between two maghemite (M) layers, i.e., $\mathrm{M} / \mathrm{BSHF} / \mathrm{M}$. 


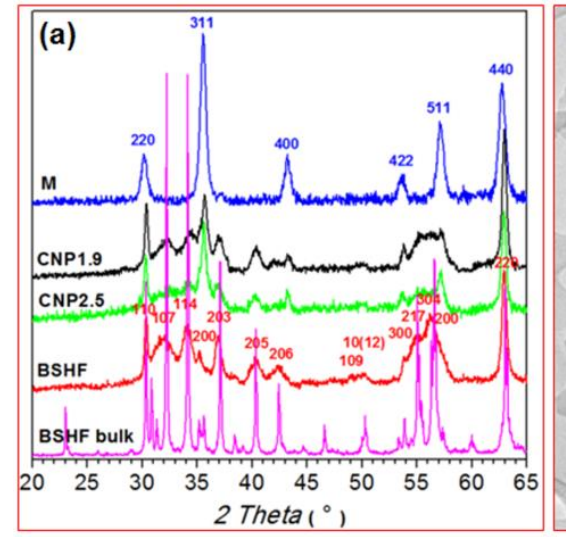

(b)

(e)
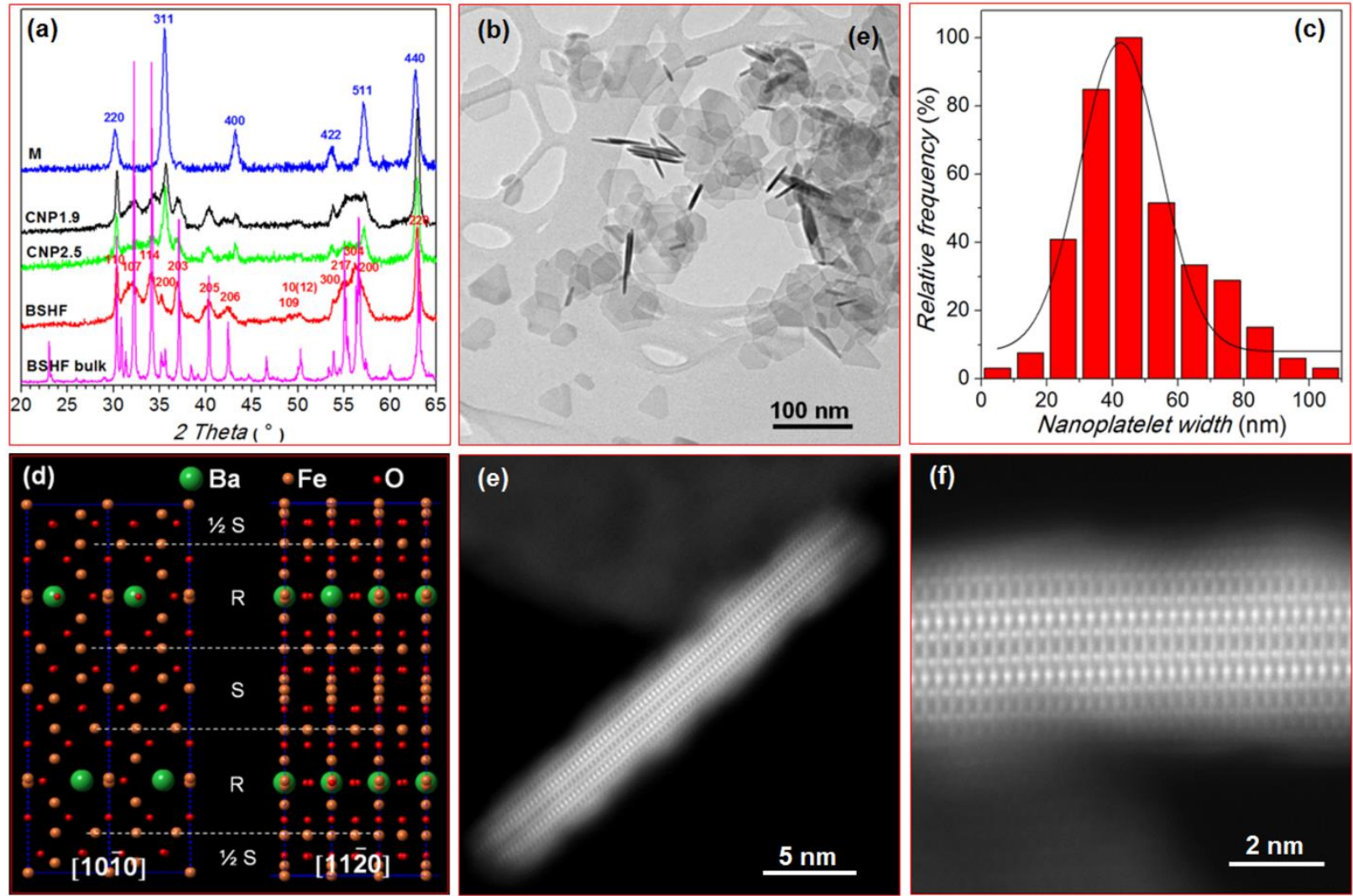

Fig. 1 XRD patterns (a) of BSHF bulk, BSHF nanoplatelets, composite nanoplatelets CNP2.5 and CNP1.9, and maghemite nanoparticles. The peaks are indexed according to the hexagonal structure of barium hexaferrite (red indexes) and the cubic spinel structure of maghemite (blue indexes). TEM image (b) of the BSHF nanoplatelets, and the corresponding distribution of the width of the nanoplatelets (c) measured from TEM images. Schematic representation of the hexaferrite structure (d). HAADF STEM images ((e) and (f)) of a BSHF nanoplatelet oriented edge-on, with the hexaferrite structure along $<11-20>$.

The thickness of the deposited M surface layers on the BSHF core nanoplatelets was successfully controlled from $1.1 \pm 0.1$ $\mathrm{nm}$ (sample CNP1.1; Fe/HF molar ratio of 2.7) to $1.5 \pm 0.1 \mathrm{~nm}$ at $\mathrm{Fe} / \mathrm{HF}$ of 3.6 (CNP1.5), and finally, to $1.9 \pm 0.2 \mathrm{~nm}$ at $\mathrm{Fe} / \mathrm{HF}$ of 5.4 (CNP1.9, Fig. 2(b)). However, when the Fe/HF ratio was increased above 5.4 the supersaturation of the precipitating iron species was too high and individual large maghemite nanoparticles formed (see ESI Fig. S3). To further increase the thickness of the surface layers, while maintaining the homogeneity of the sample, the coating process had to be conducted in two steps using the CNP1.9 nanoplatelets as the cores. The surface layers using the two-step process could be increased to be $2.5 \pm 0.2 \mathrm{~nm}$ thick (CNP2.5, Fig. 2(c)). Note that only very seldom were asymmetric composite platelets with much thicker maghemite layers on one side of the core nanoplatelet also found in the CNP2.5 sample (see ESI Fig. S4). To reveal the structural details of the composite nanoplatelets, they were further examined using atomic-resolution $\mathrm{C}_{\mathrm{S}^{-}}$ corrected STEM. In Fig. 3 the experimental HAADF image is compared with the calculated images ${ }^{45}$ based on the $\mathrm{Ba}$ hexaferrite and spinel maghemite atomic models. The positions of the cations in the projected structures (HF along $[10-10]_{\mathrm{HF}}$ and $\mathrm{M}$ along $\left.[101]_{\mathrm{M}}\right)$ are superimposed over the experimental and simulated images of Fig. 3 . Since the intensity of the spot representing an individual atomic column in the HAADF images depends on the column's average atomic number $Z, 46$ the brightest spots in the "Z-contrast" images represent the columns containing $\mathrm{Ba}^{2+}$ ions $(Z=137)$, the intensity of the columns containing the lighter $\mathrm{Fe}^{3+}$ ions $(Z=$ $56)$ is weaker and varies with their occupancy, while the $\mathrm{O}^{2-}$ columns $(Z=16)$ are not visible at all. In the hexaferrite structure the $\mathrm{Ba}^{2+}$ ions are situated in the middle of the hexagonal $\left(\mathrm{BaFe}^{3+}{ }_{6} \mathrm{O}^{2-}{ }_{11}\right)^{2-} \mathrm{R}$ block, while the $\mathrm{Fe}^{3+}$ ions occupy five different crystallographic sites, i.e., tetrahedral $\left(4 f_{1}\right)$, octahedral $\left(12 k, 2 a, 4 f_{2}\right)$, and trigonal (2b) (the different positions of the $\mathrm{Fe}^{3+}$ sites are marked in Fig. 3). In the HAADF images of the BSHF core of the composite nanoplatelets, the Ba columns alternate with Fe $2 \mathrm{~b}$ columns, making the row in the middle of the three-oxygen-layer $\mathrm{R}$ block. The mixed $\mathrm{Ba} / \mathrm{Fe}$ $2 \mathrm{~b}$ row is sandwiched between the rows of Fe $4 f_{1}$ columns. The row of Fe $12 \mathrm{k}$ columns is at the transition from the $\mathrm{R}$ block to the two-oxygen-layer cubic $\left(\mathrm{Fe}^{3+}{ }_{6} \mathrm{O}^{2-}{ }_{8}\right)^{2+} \mathrm{S}$ block. The occupancy of each second column in the Fe $12 \mathrm{k}$ row is doubled, resulting in the spots of alternating brightness. The BSHF core always terminates with a layer of $\mathrm{Fe}^{3+}$ ions at the $12 \mathrm{k}$ lattice sites, i.e., with the complete $S$ block of the hexaferrite structure. The BSHF core platelets are very uniform, containing only two $\mathrm{R}$ blocks (Fig. 2(d) and (e), Fig. 3), or seldom three R blocks (Fig. $2(\mathrm{f})$ ). Hence, the thickness of the core is either $\sim 2.9 \mathrm{~nm}$ (for the two $\mathrm{R}$ blocks) or occasionally $\sim 4.1 \mathrm{~nm}$ (for the three $\mathrm{R}$ blocks). 

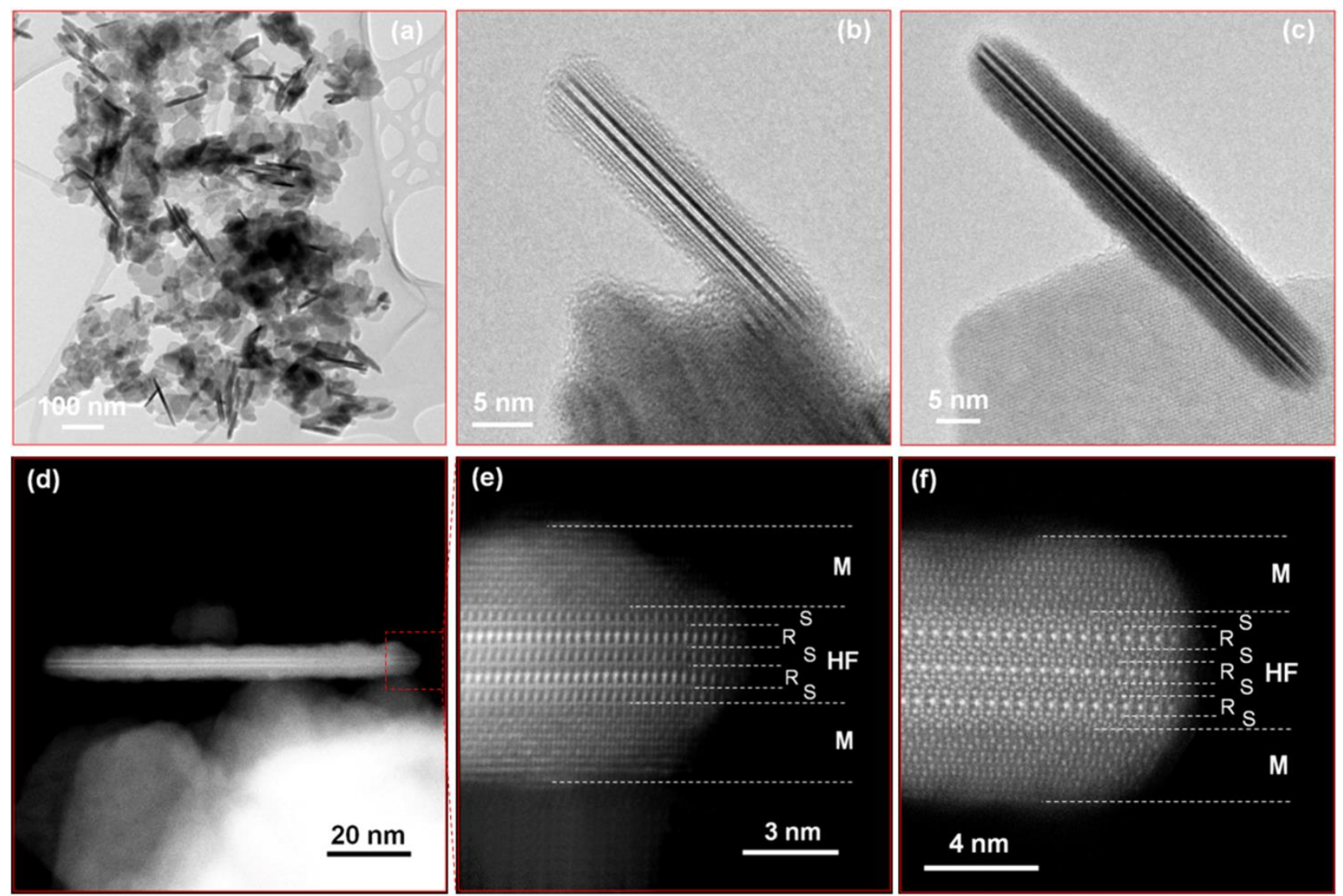

Fig. 2 TEM image of the CNP1.9 composite nanoplatelets (a). HREM images of the CNP1.9 and CNP2.5 composite nanoplatelets oriented edge on, with the basal surfaces parallel to the electron beam (b) and (c), respectively. HAADF STEM images of the CNP2.5 composite nanoplatelets with the hexaferrite (HF) core oriented along $<11-20>_{\text {HF }}((\mathrm{d})$ and $(\mathrm{e}))$ and along $<10-10>_{H F}(\mathrm{f})$.

For the M/BSHF/M composite nanoplatelets, the maghemite surface layers grow epitaxially at both the basal surfaces of the BSHF core nanoplatelets. The interface between the BSHF core and the $M$ surface layers was always atomically flat. In addition, the $M$ layers were always of uniform thickness and, in fact, the thickness of the layers on each of the basal surfaces of the cores platelet was usually equal. Given the S-block termination of the BSHF platelets, the M and BSHF structures match almost perfectly at the interface $\left((0001)_{B S H F} \|(111)_{M}\right)$. Note that a significant amount of the maghemite was never observed deposited onto the side surfaces of the core platelets, where the two structures do not match (ESI, Fig. S5). The deposition of the maghemite exclusively on the basal surfaces of the core nanoplatelets can be explained by the lower activation energy required for the heterogeneous nucleation on the S-block-terminated basal surfaces, where the two structures closely match, compared to the nucleation on the side surfaces, where the two structures do not match. Because a low supersaturation of the precipitating iron species was maintained during the deposition process, the deposit only heterogeneously nucleated on the basal surfaces, while the supersaturation was too low to trigger the nucleation on the side surfaces of the BSHF nanoplatelets.

In the $\mathrm{AB}_{2} \mathrm{O}_{4}$ spinel structure cations are distributed among tetrahedral (A) and octahedral (B) lattice sites (the positions of $\mathrm{Fe}^{3+}$ sites are marked in Fig. 3). In the structure of maghemite the (A) sites are fully occupied by $\mathrm{Fe}^{3+}$, while one-third of the (B) sites remains unoccupied (structural formula: $\left(\mathrm{Fe}^{3+}\right)\left[\mathrm{Fe}^{3+}{ }_{5 / 3} \square_{1 / 3}\right] \mathrm{O}_{4}, \square$ stands for a vacancy and the round and square brackets represent the tetrahedral and octahedral lattice sites, respectively). ${ }^{17,39}$ Since the hexaferrite structure terminates with the S block, which actually represents a (111) slice of the spinel structure, the hexaferrite structure of the core could simply extend by the $M$ spinel surface layer. However, this was never observed. Namely, the structure of the $M$ layer was always slightly shifted along the interface, making a discontinuity between the outer-most $\mathrm{S}$ layer of the core and the $\mathrm{M}$ layer. Moreover, the structural order in the $\mathrm{M}$ layers seems to be somewhat worse than in the core. 


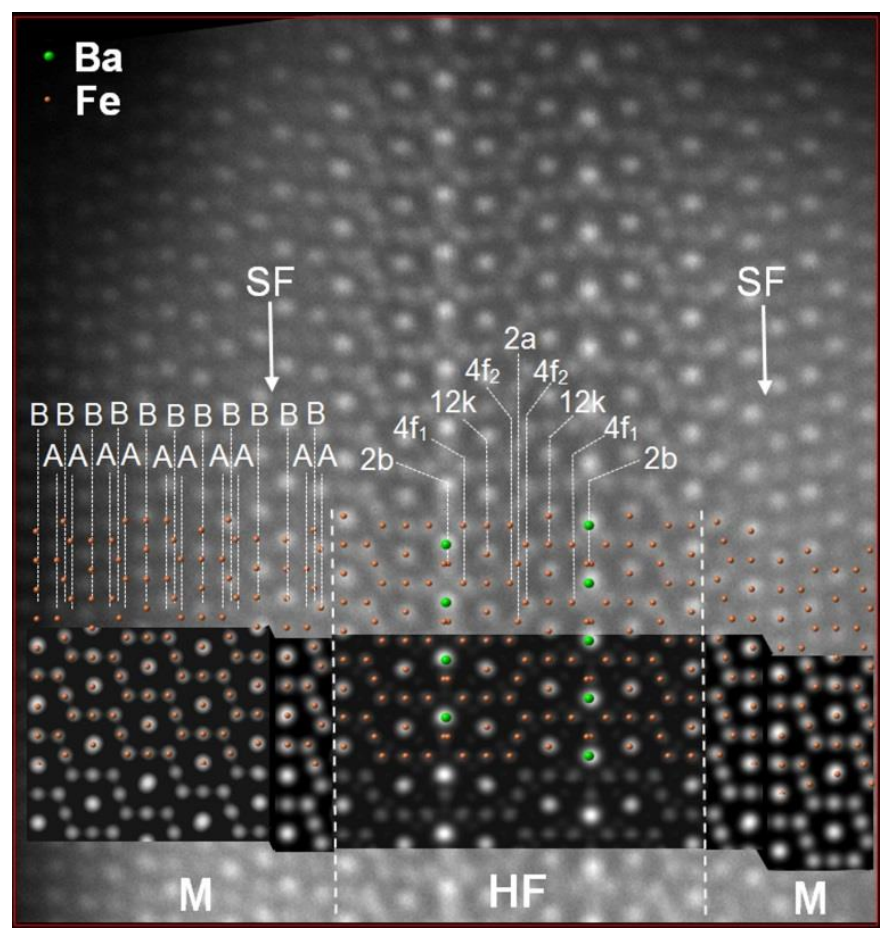

Fig. 3 HAADF STEM image of composite nanoplatelet (sample CNP2.5) with superimposed calculated images for hexaferrite (HF) along [10-10] $\mathrm{HF}$ and maghemite (M) along $[101]_{\mathrm{M}}$. The projected structures are superimposed over the image to illustrate the positions of the $\mathrm{Ba}^{2+}$ and $\mathrm{Fe}^{3+}$ ions. Different $\mathrm{Fe}$ lattice sites in the maghemite spinel structure (tetrahedral A and octahedral B) and in the hexaferrite structure (trigonal $2 \mathrm{~b}$, tetrahedral $4 \mathrm{f}_{1}, \mathrm{octahedral} 12 \mathrm{k}, 2 \mathrm{a}$, and $4 \mathrm{f}_{2}$ ) are marked. Stacking faults (marked with SF) are sometimes present in the (111) planes of the M layers.

From the STEM analysis of the BSHF cores in the composite nanoplatelets, it can be concluded that the pure BSHF platelets actually have a distinct structure that is different from the bulk hexaferrite structure, as can be indirectly inferred from the XRD and Raman studies. The bulk hexaferrite structure can be represented in terms of periodic stacking of the structural blocks with a SRS*R* unit cell (*denotes rotation over $180^{\circ}$ around the c-axis). ${ }^{17,18}$ Thus, for the nanoplatelets containing the two $\mathrm{R}$ blocks the overall structure along the c-direction exceeds the one hexaferrite unit cell by just one surface $S$ block. The whole structure of the nanoplatelet contains three $\left(\mathrm{Fe}_{6} \mathrm{O}_{8}\right)^{2+} \mathrm{S}$ blocks and two $\left(\mathrm{BaFe}_{6} \mathrm{O}_{11}\right)^{2-} \mathrm{R}$ blocks. The SRS*R*S stack, which represents the overall structure of the nanoplatelet, corresponds to a theoretical composition of $\left(\mathrm{Ba}_{2} \mathrm{Fe}_{30} \mathrm{O}_{46}\right)^{2+}$. In principle, this stacking should display a positive charge due to the additional unpaired $S$ block. As additional oxygens cannot be accommodated into the closepacked structure, the extra charge can be compensated by the reduction of $\mathrm{Fe}^{3+}$ to $\mathrm{Fe}^{2+}$ or by the formation of charged vacancies in the $\mathrm{Fe}^{3+}$ sub-lattice. In the case of the nanoplatelets containing the three $\mathrm{R}$ blocks the stacking is SRS*R*SRS*, corresponding to the theoretical composition of $\mathrm{BaFe}_{14} \mathrm{O}_{22}$.

The deviation from the $\mathrm{BaFe}_{12} \mathrm{O}_{19}$ bulk composition due to the termination of the nanoplatelet structure with the Fe-rich $\mathrm{S}$ blocks was confirmed by using quantitative energy-dispersive $\mathrm{X}$-ray spectroscopy (EDXS) analysis. The composition measured on a large number of individual nanoplatelets was compared to the composition of larger, hydrothermally synthesized, hexaferrite platelet crystals $(0.5-2 \mu \mathrm{m}$ wide and $10-20 \mathrm{~nm}$ thick, see ESI). The $(\mathrm{Fe}+\mathrm{Sc}) / \mathrm{Ba}$ molar ratio of $15.2 \pm 1.4$ measured for the nanoplatelets is significantly higher than in the larger platelet crystals $((\mathrm{Fe}+\mathrm{Sc}) / \mathrm{Ba}=12 \pm 0.9)$, matching well with the theoretical nanoplatelet composition of $\mathrm{Ba}_{2}(\mathrm{Fe}, \mathrm{Sc})_{30} \mathrm{O}_{46}$.

As the nanoplatelets display a distinct structure and composition, which are both significantly different from the bulk, they can be considered as a novel structural variation of the hexaferrites stabilized on the nanoscale. Note that although the theoretical composition of the nanoplatelets containing two $\mathrm{R}$ blocks is equal to the composition of the $\mathrm{Fe}_{2}$-X-polytype compound $\mathrm{Ba}_{2} \mathrm{Fe}^{2+}{ }_{2} \mathrm{Fe}^{3+}{ }_{28} \mathrm{O}_{46}$, the stacking of the structural blocks in the $\mathrm{Fe}_{2}-\mathrm{X}$ compound is different (RSRSSR*S*R*S*S*) and its unit cell is much larger ( $c=8.41$ $\mathrm{nm}$ ) than the thickness of the nanoplatelet. ${ }^{18}$

To explain the reason for the experimentally observed S-blocktermination, we performed ab-initio simulations, $47-49$ comparing the total energies, $E_{\text {tot }}$, for several $S$ - and Rterminated structures consisting of a fixed number of $\mathrm{Ba}$, Fe and $O$ atoms (see ESI for details). An example of the two resulting structures is presented in Fig. S7. In contrast to the experimental evidence, it was found that the surface layer of the energetically most stable structure corresponded to the $\mathrm{R}$ block. This hints that the observed novel structure is probably metastable and highlights the crucial role of the aqueous suspension and the synthesis method for the structure of the BSHF nanoplatelets, where the nanoplatelets are formed under hydrothermal conditions in the presence of a large amount of $\mathrm{NaOH}$, hydrating the surfaces. Moreover, the higher solubility of the $\mathrm{BaO}$ in water compared to the low solubility of $\mathrm{Fe}_{2} \mathrm{O}_{3}$ could also contribute to the termination of the hexaferrite nanoplatelets with the $\mathrm{S}$ block. 
Magnetic properties of hexaferrite and composite nanoplatelets.

The BSHF nanoplatelets are strongly ferromagnetic at room temperature despite their small size (Fig. 4(a)). They exhibit a moderate $\boldsymbol{M}_{\mathbf{s}}$ of about $\boldsymbol{M}_{\mathbf{s}}=30 \mathrm{emu} / \mathrm{g}$, which is actually larger than that typically observed in nanostructured Ba-ferrites of similar sizes, $16,19,20,42$ probably due to the incorporation of Sc in the structure ${ }^{21}$ and their Fe-rich composition. Moreover, the nanoplatelets are relatively magnetically hard, having a sizable coercivity of $\boldsymbol{H}_{\mathrm{C}}=1200$ Oe when randomly oriented, which indicates that this novel Ba-Sc-hexaferrite structure should have a fairly high anisotropy. To further elucidate the properties of the nanoplatelets we carried out magnetic measurements on oriented nanoplatelets (Fig. 4(a)). When measuring perpendicular (PER) to the basal plane of the platelet, i.e., along the c-axis, the loops are rather square (i.e., high remanence-to-saturation-magnetization ratio, $\boldsymbol{M}_{\mathbf{R}} / \mathbf{M}_{\mathbf{S}} \sim 1$; Fig. 4(a)), with a high coercivity. On the other hand, when measuring in the plane of the platelets (PAR) the loops show a considerably smaller $\boldsymbol{M}_{\mathrm{R}} / \boldsymbol{M}_{\mathrm{S}}$ and $\boldsymbol{H}_{\mathrm{C}}$ (Fig. 4(a)). Interestingly, this implies that the easy axis of the BSHF nanoplatelets is along the c-axis (i.e., perpendicular to the basal plane of the platelets), in concordance with bulk Ba-hexaferrite. The temperature dependence of the magnetization (Fig. 4(b), see ESI for details) shows that the magnetization decreases monotonically, vanishing at about $\mathbf{T}_{\mathbf{C}} \sim 340{ }^{\circ} \mathrm{C}$. The rather homogenous $M(T)$ indicates that no structural transition or strong growth of the nanoplatelets takes place, and $T_{C}$ is lower than for pure bulk barium hexaferrite (with $\mathbf{T}_{\mathrm{c}} \sim 450^{\circ} \mathrm{C}$ ) ${ }^{50}$. This can be explained by size effects ${ }^{42}$ or due to the partial substitution of $\mathrm{Fe}^{3+}$ with nonmagnetic $\mathrm{Sc}^{3+}$, which is also known to decreases $\mathbf{T}_{\mathrm{c}}{ }^{50}$

The deposition of the soft-magnetic maghemite layers onto the hexaferrite nanoplatelet cores leads to an effective enhancement of the overall $\boldsymbol{M}_{\mathbf{s}}$ (Fig. 4(c)). The $\boldsymbol{M}_{\mathbf{s}}$ of the composite nanoplatelets $\left(\boldsymbol{M}_{\mathbf{S}(\mathbf{C N P})}\right)$ increased in proportion to the thickness of the maghemite layers, leading to an $\boldsymbol{M}_{\mathbf{s}}$ as high as $52 \mathrm{emu} / \mathrm{g}$ (Table 1 ). Considering that $\boldsymbol{M}_{\mathbf{s} \text { (CNP) }}=\boldsymbol{w}_{\text {core }}$. $\boldsymbol{M}_{\mathbf{s} \text { (core) }}+\boldsymbol{w}_{\text {layer }} \cdot \boldsymbol{M}_{\mathbf{s} \text { (layer), the }} \boldsymbol{M}_{\mathbf{s} \text { (layer) }}$ can be estimated (mass fractions $\boldsymbol{w}_{\text {core, }} \boldsymbol{w}_{\text {layer }}$ were estimated from TEM analysis (Table 1)). The estimated values for $\boldsymbol{M}_{\boldsymbol{s} \text { (layer) }}$ increase with the thickness of the maghemite layer from $52 \mathrm{emu} / \mathrm{g}$ to $68 \mathrm{emu} / \mathrm{g}$ (Table 1), which are rather realistic values for nanosized maghemite. Note that spherical maghemite nanoparticles of a comparable iron oxide volume to CNP1.9 (i.e., $9 \mathrm{~nm}$ in diameter), synthesized by co-precipitation of $\mathrm{Fe}^{2+} / \mathrm{Fe}^{3+}$ ions, displayed an $\boldsymbol{M}_{\mathbf{s}}$ of $\sim 66 \mathrm{emu} / \mathrm{g}$ (ESI, Fig. S9).

The composite nanoplatelets displayed single-phase magnetic loops, proving that the two magnetic materials - the hardmagnetic hexaferrite and the soft-magnetic maghemite - are rigidly exchange coupled, as expected from their sizes. ${ }^{23-25}$ The presence of any significant amount of non-coupled maghemite would lead to a characteristic constricted shape for the hysteresis loop (see ESI, Fig. S9). Slightly constricted hysteresis loops were only measured for the composite CNP2.5 nanoplatelets (Fig. 4(c)). The critical thickness below which the soft phase is rigidly coupled to the hard phase is generally considered to be roughly twice the width of a domain wall in the hard phase. ${ }^{23,24}$ In bulk the width of the domain walls for Ba-ferrite is approximately $14 \mathrm{~nm},{ }^{25}$ hence much thicker than the thickness of the maghemite layers of the CNP2.5 platelets. Thus, the observed constriction could, in principle, be explained by the presence of a small quantity of uncoupled soft nanoparticles in the CNP2.5 sample. Close inspection of the sample with TEM, showed occasionally asymmetric composite nanoplatelets with much thicker maghemite layer (several tens of $\mathrm{nm}$ ) deposited on one side of the core (ESI, Fig. S4), which could lead to the constricted loops.

Even though the composite nanoplatelets displayed singlephase hysteresis loops (except CNP2.5), their coercivity $\left(\boldsymbol{H}_{\mathrm{C}}\right)$ was somewhat low, ranging from 108 Oe for CPN1.9 to 277 Oe of CPN1.5, compared to the BSHF nanoplatelets (Table 1). In fact, the $\boldsymbol{H}_{\mathrm{C}}$ of the composites decreased more than we would expect for simple hard-soft exchange-coupled structures. ${ }^{23-25}$ This indicates that the reduced $\boldsymbol{H}_{\mathrm{C}}$ is probably related to changes in the magnetic properties of the BSHF core when it is coated by the epitaxial maghemite shell, where epitaxyinduced internal stresses could lead to a $\boldsymbol{H}_{\mathrm{c}}$ reduction (as shown for composite nanoplatelets based on a non-magnetic Zn-ferrite spinel; $51-53$ see ESI).

The magnetic properties of the composite nanoplatelets were also studied after orienting them in a magnetic field. Similar to the BSHF platelets, the nanocomposite nanoplatelets also exhibit a clear easy(hard) axis behaviour when the field is applied perpendicular(parallel) to the nanoplatelets' basal plane (Fig. 4(d)). Importantly, this clearly shows that an easy axis perpendicular to the basal surfaces is roughly maintained after the deposition of the $\gamma-\mathrm{Fe}_{2} \mathrm{O}_{3}$ layers. However, the $\boldsymbol{M}_{\mathbf{R}} / \boldsymbol{M}_{\mathbf{S}}$ of the nanocomposite nanoplatelets is significantly smaller than for the BSHF nanoplatelets. This implies that in the nanocomposites the alignment with the magnetic field is somewhat worse. This probably has two different origins. Firstly, due to the increased $\boldsymbol{M}_{\mathbf{s}}$ the magnetostatic interactions tend to increase, which leads to a considerable tendency to aggregation, which can strongly affect the magnetic alignment. Although steps were taken to minimize the aggregation (e.g., the nanoplatelets were coated with ricinoleic acid as the surfactant) it is difficult to completely suppress it. Additionally, it should be taken into account that while the BSHF core has an out-of-plane easy axis, the soft $\gamma-\mathrm{Fe}_{2} \mathrm{O}_{3}$ layers should have a tendency to lie in-plane (due to the shape anisotropy). Thus, the competition between these two anisotropies could result in a somewhat tilted easy axis, ${ }^{54}$ which should also affect $M_{\mathrm{R}} / \mathbf{M s}_{\mathrm{s}}$.

Interestingly, the $\boldsymbol{M}$ vs. T curve for CNP1.1, although it has a pronounced decrease at about $\mathbf{T}_{\mathbf{C}} \sim 310{ }^{\circ} \mathrm{C}, \boldsymbol{M}$ does not vanish above this temperature and remains finite even at $550^{\circ} \mathrm{C}$. The improved high-T stability of the nanocomposites, probably 

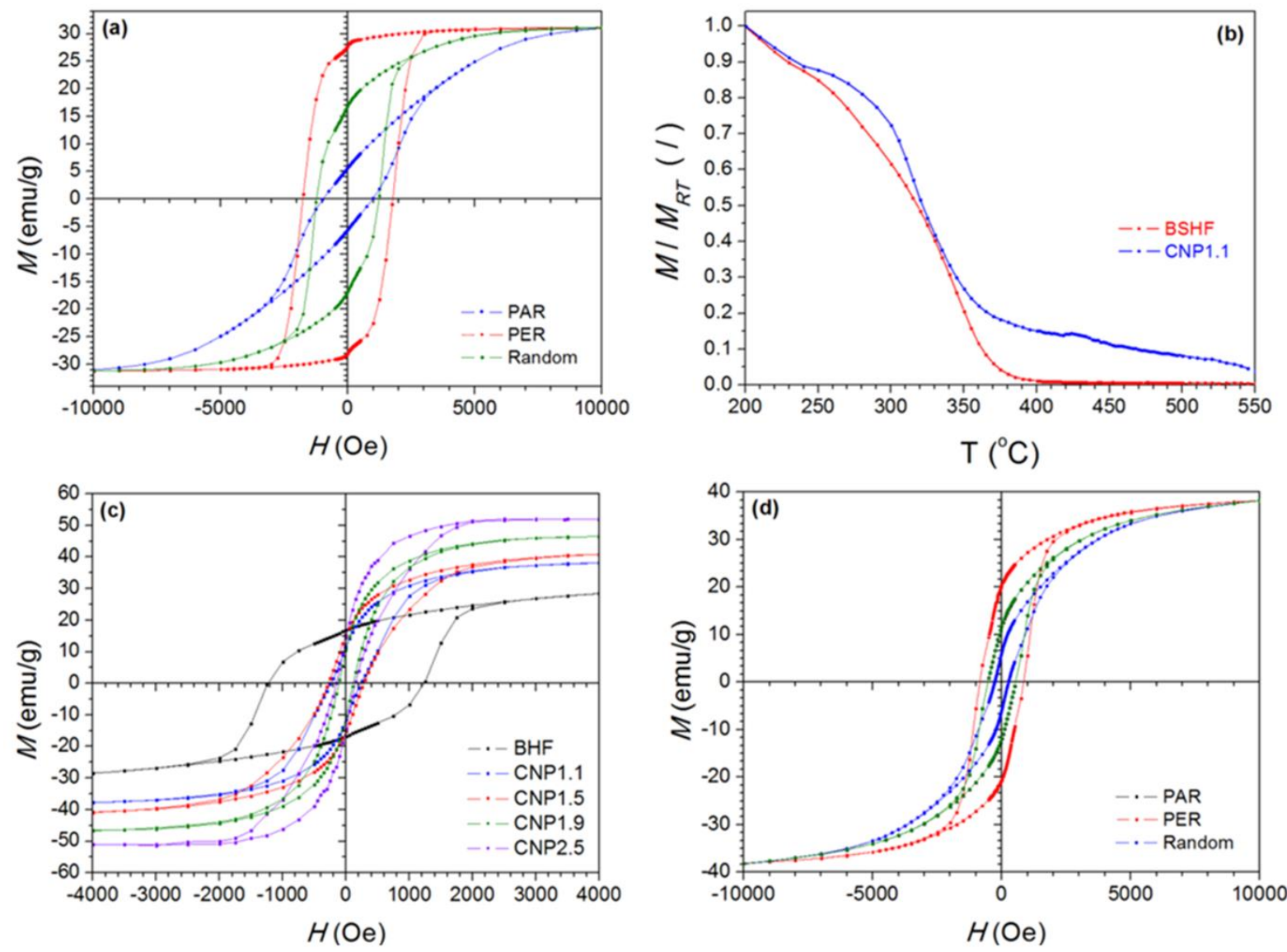

Fig. 4 Magnetic hysteresis loops for the BSHF nanoplatelets oriented randomly and perpendicular (PER) and parallel (PAR) to their basal surfaces (a). Normalized temperature dependence of the magnetization $\left(\boldsymbol{M} / \mathbf{M}_{\mathbf{R T}}\right.$ ) of the BSHF nanoplatelets and the CNP1.1 composite nanoplatelets (b). Hysteresis loops for randomly oriented BHF and CNPX.X composite nanoplatelets (c). Hysteresis loop for the aligned and randomly-oriented CNP1.1 nanoplatelets (d).

arises from the higher $\mathbf{T}_{\mathbf{c}}$ of the $\gamma-\mathrm{Fe}_{2} \mathrm{O}_{3}$ counterpart, although, observed in the composite nanoparticles upon moderate alternatively, it might be linked to the structural transition annealing (see ESI).

Table 1 Estimated composition and magnetic properties for the BHSF and composite nanoplatelets. The mass fractions $\boldsymbol{w}$ of the two phases were estimated assuming a core nanoplatelet with an average width of $42 \mathrm{~nm}$ (Fig. 1(c)) and an average thickness of $3.5 \mathrm{~nm}$.

\begin{tabular}{|c|c|c|c|c|c|c|}
\hline \multirow[t]{2}{*}{ Sample } & \multicolumn{2}{|c|}{ Estimated composition } & \multirow{2}{*}{$\begin{array}{l}M_{\mathbf{S}} \\
{[\mathrm{emu} / \mathrm{g}]}\end{array}$} & \multirow{2}{*}{$\begin{array}{l}M_{\mathbf{R}} \\
{[\mathrm{emu} / \mathrm{g}]}\end{array}$} & \multirow{2}{*}{$\begin{array}{l}\boldsymbol{H}_{\mathrm{C}} \\
{[\mathrm{Oe}]}\end{array}$} & \multirow{2}{*}{$\begin{array}{l}M_{\mathbf{S}(\text { layer) }} \\
{[\text { emu/g] }}\end{array}$} \\
\hline & $\boldsymbol{w}_{\text {core }}[/]$ & $\boldsymbol{w}_{\text {layer }}[/]$ & & & & \\
\hline BSHF & 1 & 0 & 31 & 16.8 & 1250 & / \\
\hline CNP1.1 & 0.66 & 0.34 & 38 & 11.6 & 239 & 52 \\
\hline CNP1.5 & 0.56 & 0.44 & 42 & 15.3 & 277 & 56 \\
\hline CNP1.9 & 0.50 & 0.50 & 47 & 10.5 & 108 & 63 \\
\hline CNP2.5 & 0.43 & 0.57 & 52 & 14.2 & 149 & 68 \\
\hline
\end{tabular}

\section{Conclusions}

A novel structure of Ba-hexaferrite nanoplatelets was identified by STEM that was formed by only two $\left(\left(\mathrm{BaFe}_{6} \mathrm{O}_{11}\right)^{2-}\right)$ $\mathrm{R}$ structural blocks and three $\left(\left(\mathrm{Fe}_{6} \mathrm{O}_{8}\right)^{2+}\right) \mathrm{S}$ blocks in a $\mathrm{SR}^{*} \mathrm{~S}^{*} \mathrm{RS}$ stacking sequence, corresponding to a theoretical $\mathrm{BaFe}_{15} \mathrm{O}_{23}$ formula and a thickness of $2.9 \mathrm{~nm}$. Only a minor part of the nanoplatelets displayed three $\mathrm{R}$ blocks with the SRS*R*SRS* stacking and a theoretical $\mathrm{BaFe}_{14} \mathrm{O}_{22}$ composition. These structures are stabilized by the nanoscale dimensions and the synthesis (aqueous) approach. Sandwiched composite nanoplatelets were synthesized by the growth of epitaxial maghemite layers at the basal surfaces of these hexaferrite (Sc-doped $\mathrm{BaFe}_{12} \mathrm{O}_{19}$ ) nanoplatelets using a simple, robust method based on the controlled co-precipitation of $\mathrm{Fe}^{3+} / \mathrm{Fe}^{2+}$ in the aqueous suspension. The composite nanoplatelets displayed an incredibly uniform structure, defined by the 
uniform structure of the hexaferrite cores and the homogeneous thickness of the epitaxial layers. The $\mathrm{Ba}$ hexaferrite nanoplates show a modest $\boldsymbol{M}_{\mathbf{s}}(31 \mathrm{emu} / \mathrm{g}$ ) and a rather large $\boldsymbol{H}_{\mathrm{C}}(1200 \mathrm{Oe})$ and an easy axis perpendicular to the nanoplatelet basal plane. The hysteresis loops of the composite nanoplatelets confirm that the hard/soft components are rigidly exchange coupled. Importantly, the composite nanoplatelets retain the out-of-plane easy axis of the hexaferrite cores, while substantially enhancing the saturation magnetization $M_{\mathbf{S}}(52 \mathrm{emu} / \mathrm{g}$ ), which increases in proportion to the thickness of the deposited maghemite layers. However, the increase in the $\boldsymbol{M}_{\mathbf{S}}$ is accompanied by a relative de-crease of $\boldsymbol{H}_{\mathrm{c}}$, in part related to the epitaxial strains.

The extraordinary properties of the composite structures, i.e., nanoplatelet shape, rigid exchange coupling, and out-of-plane magnetization with $M_{\mathbf{s}}>50 \mathrm{emu} / \mathrm{g}$, should enable the development of new magneto-responsive materials and applications based on the magnetic field alignment of the nanoplatelets.

\section{Conflicts of interest}

There are no conflicts to declare.

\section{Acknowledgements}

The support of the Slovenia Research Agency within the Program P2-0089 and the Project J2-8166, the Generalitat de Catalunya (2014-SGR-1015) and the Spanish Ministerio de Economía y Competitividad (MINECO; MAT2016-77391-R) is gratefully acknowledged. ICN2 acknowledges support from the Severo Ochoa Program (MINECO, SEV-2013-0295). The authors also like to thank Prof. Zvonko Jagličić for helpful discussions.

\section{References}

1 G. Ferk, P. Kranjc, A. Hamler, A. Mertelj, F. Cebollada, M. Drofenik and D. Lisjak, Sci. Rep. 2015, 5, DOI: $10.1038 /$ srep11395.

2 B. Rožič, M. Jagodič, S. Gyergyek, M. Drofenik, S. Kralj, Z. Jagličić, Z. Kutnjak, Ferroelectrics, 2012, 21, 150-153.

3 A. Mertelj, D. Lisjak, M. Drofenik and M. Čopič, Nature, 2013, 504, 237-241.

4 A. Mertelj, N. Osterman, D. Lisjak and M. Čopič, Soft Matter, 2014, 10, 9065-9072.

5 Q. Liu, P. J. Ackerman, T. C. Lubensky and I. I. Smalyukh Proc. Natl. Acad. Sci., 2016, 113, 10479-10484.

6 Q. Zhang, P. J. Ackerman, Q. Liu and I. I. Smalyukh, Phys. Rev. Lett. 2015, 115, 097802.

7 P. J. Ackerman and I. I. Smalyukh II. Nat. Mater. 2017, 16, 426-432.

8 R. Sahoo, M. V. Rasna, D. Lisjak, A. Mertelj and S. Dhara, Appl. Phys. Lett., 2015, 106, 161905.
9 Y. I. Golovin, S. L. Gribanovsky, D. Y. Golovin, N. L. Klyachko, A. G. Majouga, A. M. Master, M. Sokolsky and A. V. Kabanov, J. Controlled Release, 2015, 219, 43-60.

10 D.-H. Kim, P. Karavayev, E. A. Rozhkova, J. Pearson, V. Yefremenko, S. D. Bader and V. Novosad, J. Mater. Chem., 2011, 21, 8322-8326.

11 D.-H. Kim, E. A. Rozhkova, I. V. Ulasov, S. D. Bader, T. Rajh, M. S. Lesniak, V. Novosad, Nat. Matter., 2010, 9, 165-171.

12 P. M. Rupnik, D. Lisjak, M. Čopič and A. Mertelj, Liq. Cryst., 2015, 42, 1648-1688.

13 R. Geryak, J. Geldmeier, K. Wallace and V. V. Tsukruk, Nano Lett. 2015, 15, 2679-2684.

14 M. Mur, J. A. Sofi, I. Kvasić, A. Mertelj, D. Lisjak, V. Niranjan, I. Muševič and S. Dhara. Opt. Express, 2017, 25, 1073-1083.

15 A. Alfadhel and J. Kosel, Adv. Mater. 2015, 27, 7888-7892.

16 D. Makovec, D. Primc, S. Šturm, A. Kodre, D. Hanžel and M. Drofenik, J. Sol. State Chem., 2012, 196, 63-71.

17 J. P. Smith and H. P. J. Wjin, Ferrites: Physical Properties of Ferromagnetic Oxides in Relation to their Technical Applications, Philips' Technical Library, Eindhoven, 1959.

18 J. A. Kohn, D. W. Eckart, Jr. F. C. Charles, Science, 1971, 172, 519-525.

19 M. Drofenik, I. Ban, G. Ferk, D. Makovec, A. Žnidaršič, Z. Jagličić and D. Lisjak, J. Am. Ceram. Soc., 2010, 93, 16021607.

20 D. Primc, D. Makovec, D. Lisjak and M. Drofenik, Nanotechnology, 2009, 20, 315605-1-315605-9.

21 D. Lisjak and M. Drofenik, Cryst. Growth Des., 2012, 12, 5174-5179.

22 M. Shuai, A. Klittnick, Y. Shen, G. P. Smith, M. R. Tuchband, C. Zhu, R. G. Petschek, A. Mertelj, D. Lisjak, M. Čopič, J. E. Maclennan, M. A. Glaser and N. A. Clark, Nat.Commun., 2016, 7, 10394-1-10394-8.

23 E. Kneller and R. Hawig, IEEE Trans. Magn., 1991, 27, 35883600.

24 R. Skomski, and J. M. D.Coey, Phys. Rev., 1993, 48, 15812.

25 A. López-Ortega, M. Estrader, G. Salazar-Alvarez, A. G. Roca and J. Nogués, Physics Reports, 2015, 553, 1-32.

26 H. T. Hai, H. T. Yang, H. Kura, D. Hasegawa, Y. Ogata, M. Takahashi and T. Ogawa, J. Colloid Interface Sci., 2010, 346, 37-42.

27 B. P. Pichon, O. Gerber, C. Lefevre, I. Florea, S. Fleutot, W. Baaziz, M. Pauly, M. Ohlmann, C. Ulhaq, O. Ersen, PierronBohnes Véronique, P. Panissod, M. Drillon and S. Begin-Colin, Chem. Mater., 2011, 23, 2886-2900.

28 E. Wetterskog, C.-W. Tai, J. Grins, L. Bergström and G. Salazar-Alvarez, ACS Nano, 2013, 7, 7132-7144.

29 E. Lottini, A. Lopez-Ortega, G. Bertoni, S. Turner, M. Meledina, G. V. Tendeloo, C. D. J. Fernandez and C. Sangregorio, Chem. Mater., 2016, 28, 4214-4222.

30 A. López-Ortega, E. Lottini, G. Bertoni, C. D. J. Fernández and C. Sangregorio, Chem. Mater., 2017, 29, 1279-1289.

31 O. Masala, D. Hoffman, N. Sundaram, K. Page, T. Proffen, G. Lawes and R. Seshadri, Solid State Sci., 2006, 8, 1015-1022.

32 J.-H. Lee, J.-T. Jang, J.-S. Choi, S. H. Moon, S.-H. Noh, J.-W. Kim, J.-G. Kim, I.-S. Kim, K. I. Park and J. Cheon, Nature Nanotechnol., 2011, 6, 418-422.

33 Q. Song and Z. J. Zhang, Am. Chem. Soc., 2012, 134, 1018210190.

34 C. Binns, M. T. Qureshi, D. Peddis, S. H. Baker, P. B. Howes, A. Boatwright, S. A. Cavill, S. S. Dhesi, L. Lari, R. Kröger and S. Langridge, Nano Lett., 2013, 13, 3334-3339.

35 M. Estrader, A. López-Ortega, S. Estradé, I. V. Golosovsky, G. Salazar-Alvarez, M. Vasilakaki, K. N. Trohidou, M. Varela, D. C. Stanley, M. Sinko, M. J. Pechan, D. J. Keavney, F. Peiró, S. Suriñach, M. D. Baró and J. Nogués, Nature Commun., 2013, 4, 1-8.

36 D. Primc and D. Makovec, Nanoscale, 2015, 7, 2688-2679.

37 D. Primc, B. Belec and D. Makovec, J. Nanopart. Res., 2016, 18, 64-1-64-13. 
38 S. Asuha, S. Zhao, X. H. Jin, M. M. Hai and H. P., Bao, Appl. Surf. Sci., 2009, 255, 8897-8901.

39 R. M. Cornell and U. Schwertmann, The Iron Oxides: Structure, Properties, Reactions, Occurrences and Uses, VileyVCH, Weinheim, 2003.

40 S. Kralj, D. Makovec, S. Čampelj and M. Drofenik, J. Magn. Magn. Mater., 2010, 322, 1847-1853.

41 A. López-Ortega, M. Estrader, G. Salazar-Alvarez, S. Estradé, I. V. Golosovsky, R. K. Dumas, D. J. Keavney, M. Vasilakaki, K. N. Trohidou, J. Sort, F. Peiró, S. Suriñach, M. D. Baró and J. Nogués, Nanoscale, 2012, 4, 5138-5147.

42 J. Wang, F. Zhao, W. Wu and G.-M. Zhao, J. Appl. Phys., 2011, 110, 123909

43 J. H. Monkhorst and J. D. Pack, Phys. Rev. B., 1976, 13, 51885192.

44 I. Chamritski, G. Burns, J. Phys. Chem. B, 2005, 109, 49654968.

45 K. Koch, Determination of Core Structure Periodicity and Point Defect Density along Dislocations, Arizona State University, 2002.

46 S. J. Pennycook and P. D. Nellist, Scanning Electron Microscopy, Springer, New York, 2011.

47 Giannozzi, P., Baroni, S.,, Bonini, N., Calandra, M:, Car, R., Cavazzoni, C., D. Ceresoli, G.L. Chiarotti, M. Cococcioni, I. Dabo, A.D. Corso, S. de Gironcoli, S. Fabris, G. Fratesi, R. Gebaurer, U. Gerstmann, C. Gougoussis, A. Kokalj, M. Lazzeri, L. Martin-Samos, N. Marzari, F. Mauri, R. Mazzarello, S. Paolini, A, Pasquarello, L. Paulatto, C. Sbraccia, S. Scandolo, G. Sclauzero, A.P. Seitsonena, A. Smogunov, P. Umari and R.M. Wentzcovitch, J. Phys. Condens. Matter., 2009, 21, 395502.

48 J. P.Perdew, K. Burke and M. Ernzerhof, M. Phys. Rew. Lett., 1996, 77, 3865-3868.

49 N. Troullier and J.L. Martins, Phys. Rev. Lett., 1991, 43, 19932006.

50 G. Albanese and A. Deriu, Ceramurgia International, 1979, 5 3-10.

51 H. S. O'Neill, Eur. J. Mineral., 1992, 4, 571-580.

52 D. Makovec, A. Kodre, I. Arčon, J. Nanopart. Res., 2011, 13, 1781-1790.

53 D. Makovec and M. Drofenik, J. Nanopart. Res., 2008, 10, 131-141.

54 T. E. N. A. Nguyen, R. Knut, V. Fallahi, S. Chung, Q. T. Le, S. M. Mohseni, O. Karis, S. Peredkov, R. K. Dumas, C. W. Miller and J. Åkerman, Phys. Rev. Appl., 2014, 2, 044014-7. 


\section{Electronic Supplementary Information}

Novel Ba-hexaferrite structural variations stabilized on the nanoscale as building blocks for epitaxial bi-magnetic hard/soft sandwiched maghemite/hexaferrite/maghemite nanoplatelets with out-of-plane easy axis and enhanced magnetization

Blaž Belec, Goran Dražić, Sašo Gyergyek, Benjamin Podmiljšak, Tanja Goršak, Matej Komelj, Josep Nogués, Darko Makovec

\section{Materials}

Iron (III) nitratehepta hydrate $\left(\mathrm{Fe}\left(\mathrm{NO}_{3}\right)_{3} \cdot \mathrm{H}_{2} \mathrm{O}\right.$, iron (II) chloride $\left(\mathrm{FeCl}_{2}\right)$, magnesium hydroxide $\left(\mathrm{Mg}(\mathrm{OH})_{2}\right)$, urea $\left(\mathrm{CH}_{4} \mathrm{~N}_{2} \mathrm{O}\right)$, barium nitrate $\left(\mathrm{Ba}\left(\mathrm{NO}_{3}\right)_{2}\right)$, scandium nitrate $\left(\mathrm{Sc}\left(\mathrm{NO}_{3}\right)_{2}\right)$, sodium hydroxide $(\mathrm{NaOH})$, nitric acid $\left(\mathrm{HNO}_{3}\right)$, ammonia solution $\left(\mathrm{NH}_{3}\right.$, 25\%), polyvinilpirolidone (PVP) were purchased from Alfa Aesar. A nitrate complex $\mathrm{Fe}^{3+}$-urea $\left(\left[\mathrm{Fe}\left(\left(\mathrm{CO}\left(\mathrm{NH}_{2}\right)_{2}\right)_{6}\left(\mathrm{NO}_{3}\right)_{3}\right]\right)\right.$, was synthesized according to the procedure described in the literature. ${ }^{\mathrm{S} 1}$

For comparison with the Sc-doped hexaferrite (BSHF) nanoplatelets the bulk Ba-Sc ceramics (BSHF bulk) were prepared by sintering the nanoplatelets. The nanoplatelets were pressed into compacts and sintered at $1200{ }^{\circ} \mathrm{C}$ for one hour (with heating and cooling rates of $20^{\circ} \mathrm{C} \mathrm{min}^{-1}$ ).

\section{Characterization}

\section{X-ray diffractometry}

The X-ray diffractometry (XRD) of the nanoparticles was performed using a PANalytical X'Pert PRO diffractometer.

\section{Raman spectroscopy}

Raman spectra of powdered samples were recorded with a NT-MDT model Integra Spectra for Materials Science, equipped with a confocal microscope, at room temperature. The $488 \mathrm{~nm}$ laser diode was used as excitation. The scattered light was detected by a cooled CCD camera and a 600 grooves $\mathrm{mm}^{-1}$ grating. The power density was approx. $5 \mathrm{~mW}$. To obtain good signal-to-noise ratio and to prevent sample damage 
several measuring conditions were tested. The first spectrum was acquired for $10 \mathrm{~s}$, then the time was increased to $60 \mathrm{~s}$ and finally 10 spectra measured for $60 \mathrm{~s}$ were accumulated. At final spectrum at the same position was acquired for $60 \mathrm{~s}$. The same procedure was repeated at different locations and the spectra were compared. Importantly, the spectra differ only in signal-to-noise ratios and absolute values of intensities, while the positions of bands and their relative intensities were always the same. The presented spectra were obtained by accumulating 10 spectra each acquired for $60 \mathrm{~s}$, since they showed best signal-to-noise ratios.

\section{Electron microscopy}

For transmission (TEM) and scanning-transmission (STEM) electron microscopy studies, the nanoplatelets were suspended in ethanol and deposited on a copper-gridsupported lacy carbon foil. The TEM analyses were performed using a field-emission electron-source TEM Jeol 2010F equipped with an Oxford Instruments ISIS300 energy dispersive X-ray spectroscopy (EDXS) detector operated at $200 \mathrm{kV}$.

For the STEM analyses a probe Cs-corrected Jeol ARM 200CF STEM was operated at $80 \mathrm{kV}$. During the analysis of the samples, HAADF and ABF detectors were used simultaneously at 68-180 and 10-16 mrad collection semi angles, respectively. To minimize the specimen drift, images were taken several hours after the insertion of the sample in the microscope and at least 20 minutes after the last sample positioning to minimize the goniometer drift. The chemical composition was analyzed using a Jeol Centurio EDXS system with $100 \mathrm{~mm}^{2}$ SDD detector and Gatan GIF Quantum ER Dual EELS system.

The simulated HAADF STEM images were calculated based on the atomic models for barium hexaferrite and maghemite and the microscope parameters using the quantitative STEM (QSTEM) code with a multi-slice method and frozen phonon approximation. ${ }^{\text {S2 }}$

\section{Magnetic measurements}

The room-temperature magnetization $\boldsymbol{M}$ as a function of the magnetic field $\boldsymbol{H}$ of the samples was measured using a Lake Shore 7307 vibrating-sample magnetometer (VSM) with a maximum applied filed of $10 \mathrm{kOe}$. To prepare the specimens for the VSM measurements minimizing dipole-dipole interactions, nanoplatelets $(\sim 1 \mathrm{mg})$ were added into water $(\sim 2 \mathrm{~mL})$ under intensive stirring. Then, sucrose $(2 \mathrm{~g})$ was dissolved in the suspension. After drying, the solid was heated to $160{ }^{\circ} \mathrm{C}$ to melt the sucrose. The 
viscous suspension of nanoplatelets was rapidly cooled to solidify it. The obtained solid was milled and subsequently uniaxially pressed into cubic compacts. The magnetization of the cube compacts containing the nanoplatelets were measured with the magnetic field applied in three normal directions of the cube (along the pressing direction (x-axis) and perpendicular to the pressing direction (y-axis, z-axis)) using the VSM. The three measurements were averaged to obtain the magnetic properties of the randomly-oriented nanoplatelets.

To orient the nanoparticles for perpendicular and parallel magnetic measurements, the nanoplatelets were first hydrophobized by the adsorption of ricinoleic acid onto their surfaces and then a low concentration of nanoparticles was homogeneously dispersed in a liquid wax heated to $80{ }^{\circ} \mathrm{C}$. While at $80{ }^{\circ} \mathrm{C}$ a homogeneous magnetic field of $10 \mathrm{kOe}$ was applied and maintained while the wax was solidified by cooling.

The same VSM device was used for the temperature dependent measurements. Note that the nanoplatelets were coated with a silica shell prior to the measurements to minimize growth and excessive agglomeration at high temperatures. For the deposition of the silica shell onto the nanoplatelets a modified Stober process was used, as described in ref. S3.

The high temperature measurements where conducted under an argon atmosphere and a constant applied magnetic field of 200 Gauss. The magnetization was measured at different temperature intervals with a heating rate of $5{ }^{\circ} \mathrm{C} \mathrm{min}-1$ with a 1 min settling time. The Curie temperature was calculated from the thermomagnetic data, by finding the minimum of its temperature derivative. 


\section{Properties of hexaferrite nanoplatelets}

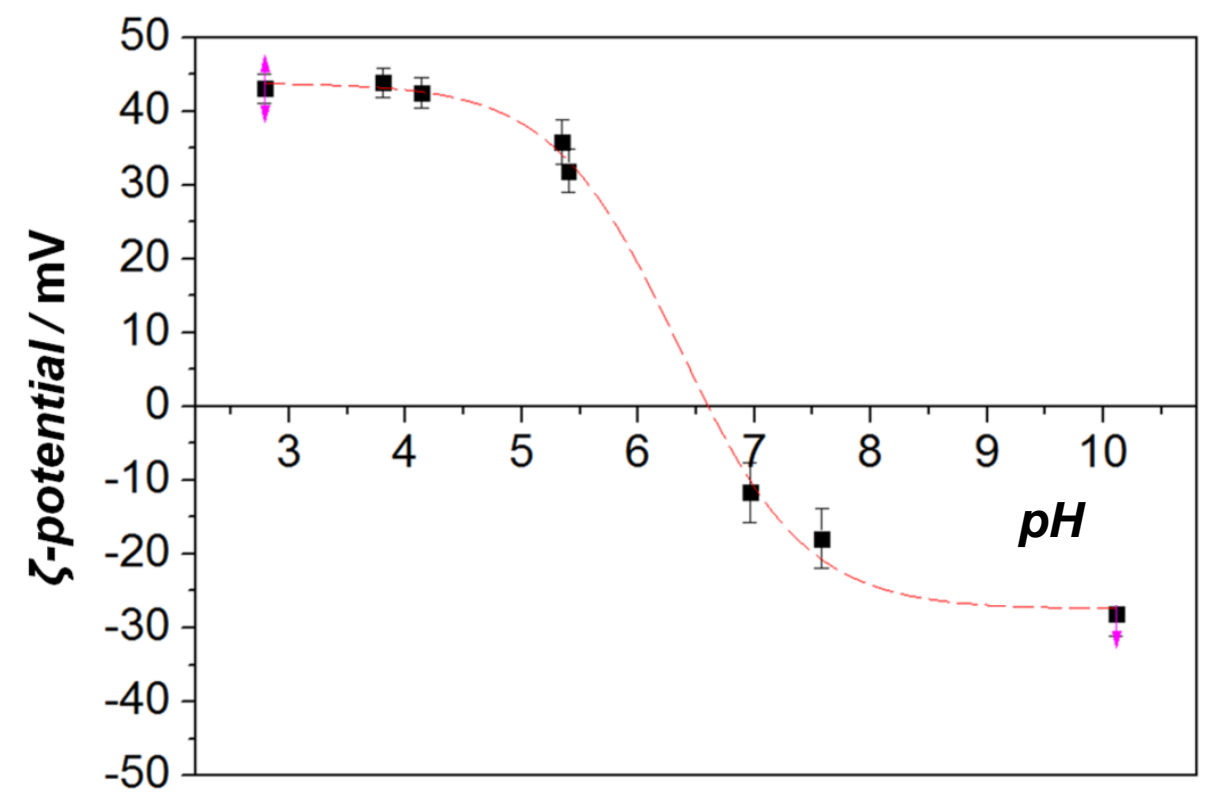

Fig. $\mathbf{S 1} \zeta$-potential of the BSHF nanoparticles as a function of the suspension $\mathrm{pH}$.

\section{Raman spectroscopy analysis of the composite nanoplatelets}

Barium hexaferrite has a rich spectrum with 42 Raman active modes. ${ }^{\mathrm{S}}$ All of the bands present in the spectrum of BSHF nanoplatelets (Fig. S2) are characteristic for the barium hexaferrite structure. ${ }^{\mathrm{S} 4}$ However, after sintering the nanoplatelets into the ceramics two additional weak bands appeared at $228 \mathrm{~cm}^{-1}$ and $298 \mathrm{~cm}^{-1}$. These two spectra can be ascribed to the presence of hematite, ${ }^{\mathrm{S} 5, \mathrm{~S} 6}$ which appeared in the ceramics due to deviation of the BSHF nanoplatelets structure and the composition from the bulk composition.

The Raman spectrum of maghemite nanoparticles $M$ shows bands at positions characteristic of iron oxide having a maghemite structure. ${ }^{\mathrm{S} 5, \mathrm{~S} 6}$ There are three Raman active lattice vibrations that give rise to three bands having a broad structure. ${ }^{\mathrm{S} 6}$ The distinction between maghemite and magnetite, which shows richer Raman spectra is in position of the bands, which are in case of magnetite shifted to lower wavenumbers by at least $30 \mathrm{~cm}^{-1}$. SI6 The difference in bands position for the maghemite and the magnetite is significantly larger than spectral resolution. Composite nanoplatelets CNP1.9 show additional band at approximately $380 \mathrm{~cm}^{-1}$ which is absent in the spectrum of the BSHF 
and is not characteristic of the barium hexaferrite structure. Additional band is very close to the maghemite characteristic band found in the spectrum for maghemite nanoparticles $\mathrm{M}$ positioned at $350 \mathrm{~cm}^{-1}$. This band is the only one in maghemite that is not overlapping with the bands of barium hexaferrite. Also in the CNP1.9 spectrum the band at approx. $300 \mathrm{~cm}^{-1}$, where the strong band is observed in magnetite, is absent. ${ }^{\mathrm{S} 6}$ We believe that this is a firm evidence that the spinel layer at the hexaferrite core of the composite nanoplatelets crystalizes in the maghemite structure.

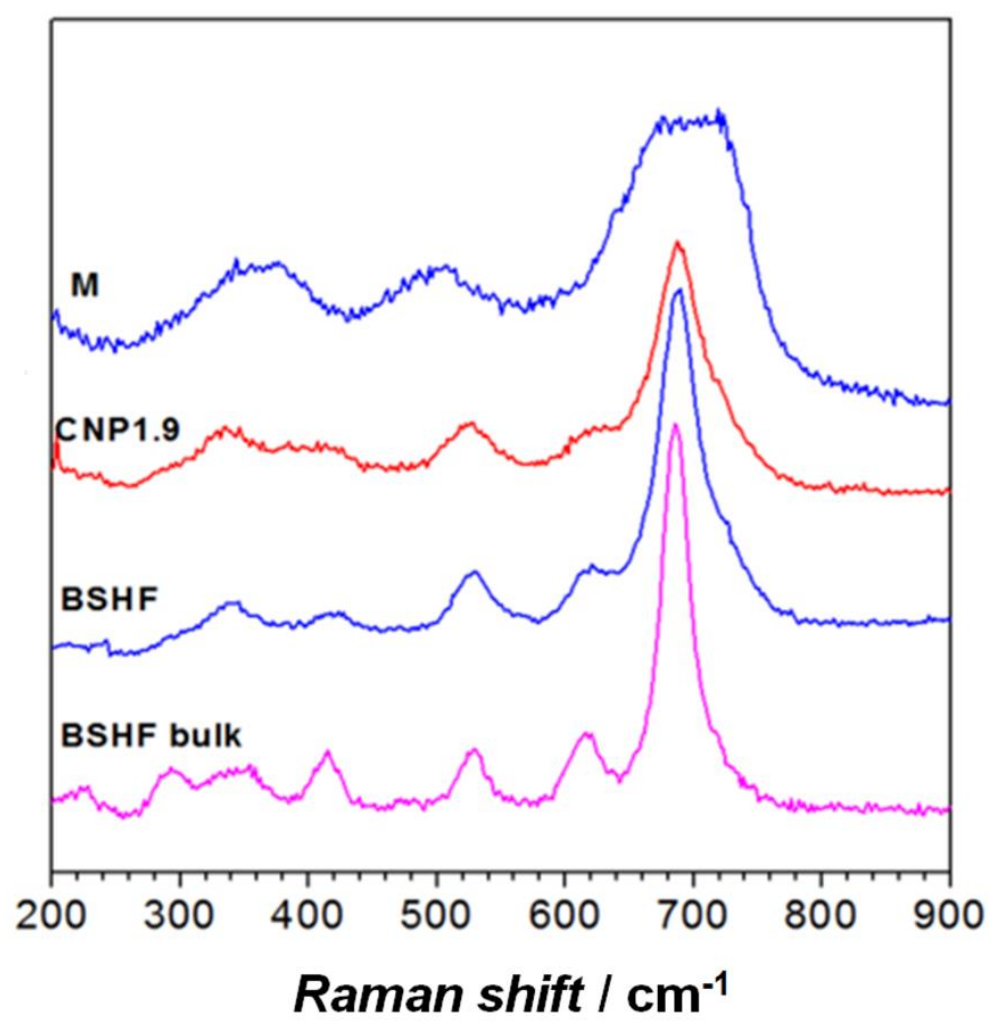

Fig. S2 Raman spectra of the maghemite nanoparticles (M), hexaferrite core nanoplatelets (BSHF), hexaferrite ceramics (BSHF bulk), and the composite nanoplatelets CNP1.9. 


\section{TEM analysis of the composite nanoplatelets synthesized using too large $\mathrm{Fe} / \mathrm{HF}$ ratio}

When the Fe/HF molar ratio exceeded 5.4 individual larger maghemite nanoparticles form in the samples. Fig. S3 shows a TEM image of the sample synthesized using the $\mathrm{Fe} / \mathrm{HF}$ molar ratio of 8.1. Some large maghemite nanoparticles are marked with arrows.

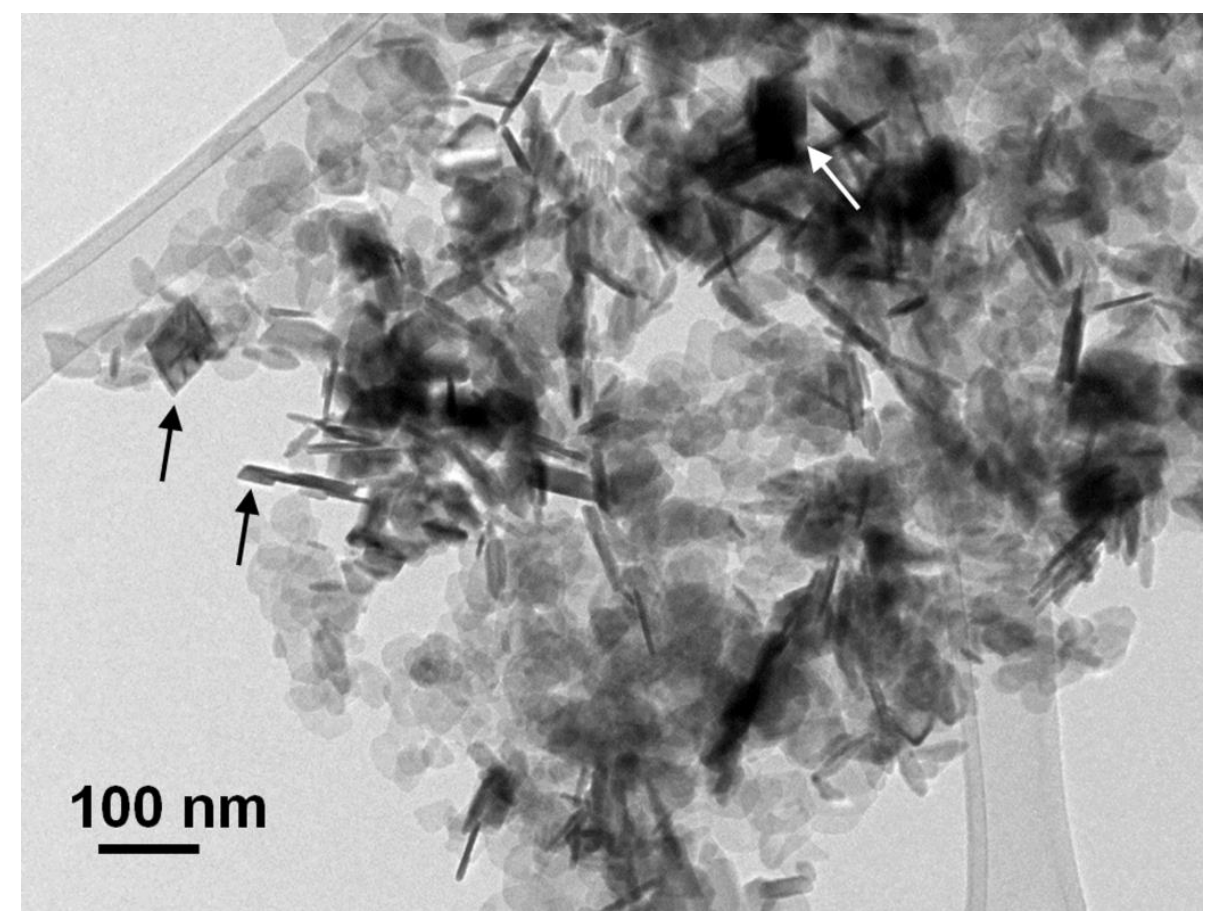

Fig. S3 TEM image of composite nanoparticles synthesized using $\mathrm{Fe} / \mathrm{HF}=8.1$. Large maghemite nanoparticles are marked with arrows. 


\section{Analysis of the composite nanoplatelets CNP2.5}

In the CNP2.5 sample synthesized by deposition of maghemite layers onto the BSHF nanoplatelets in the two-step procedure, asymmetric composite nanoplatelets were occasionally found. Fig. S4 shows HRTEM image of one such asymmetric composite nanoplatelets.
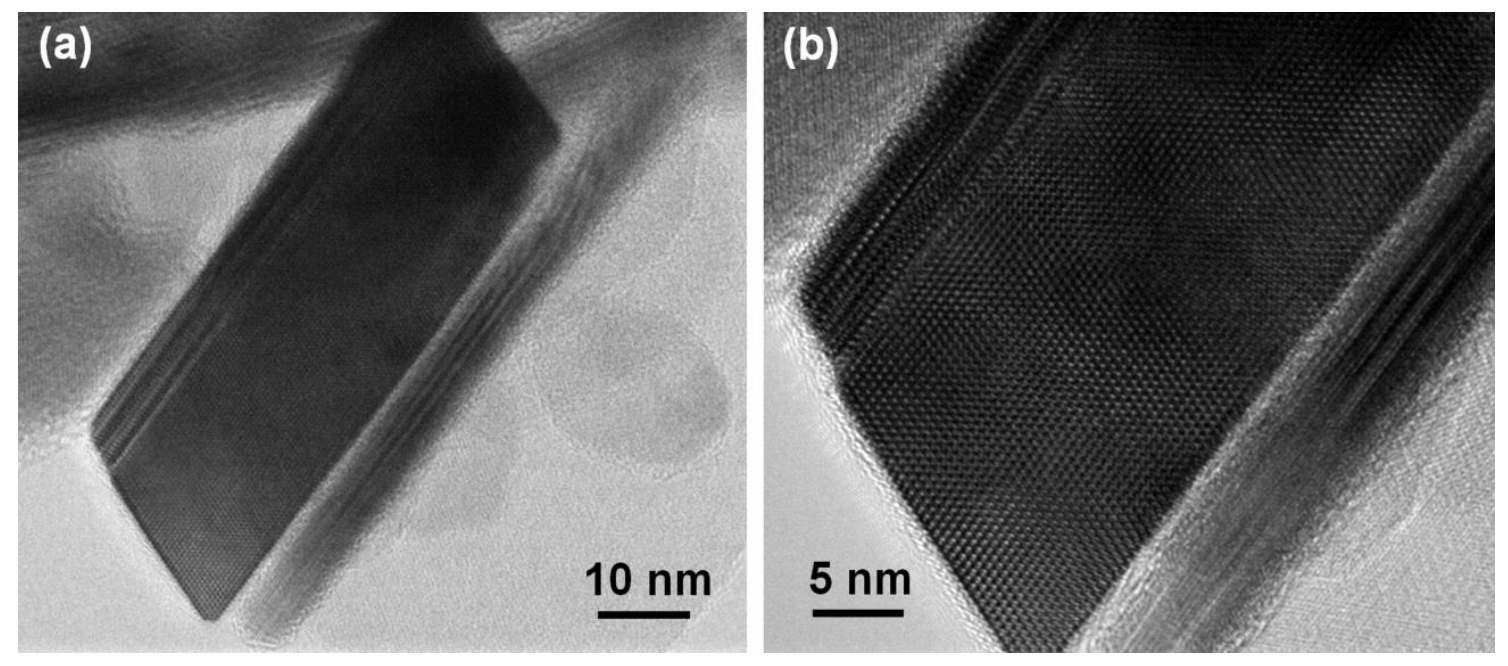

Fig. S4 HRTEM images of an asymmetric composite nanoparticle in the CNP2.5 sample $\left(\langle 10-10\rangle_{\mathrm{HF}} \|\langle 101\rangle_{\mathrm{M}}\right)$. 
Even when the composite nanoplatelets were synthesized in the two-step process (sample CNP2.5) the maghemite deposited exclusively as epitaxial layers on both the basal surfaces of the hexaferrite core nanoplatelets, while the side surfaces were always free of any deposited material. Fig. S5 shows a HAADF STEM image of the composite nanoplatelet in the sample CNP2.5. A comparison of the experimental image with the superimposed projected hexaferrite structural model shows that the atomic columns on the side of the hexaferrite core can be ascribed to the hexaferrite structure on the very surface.

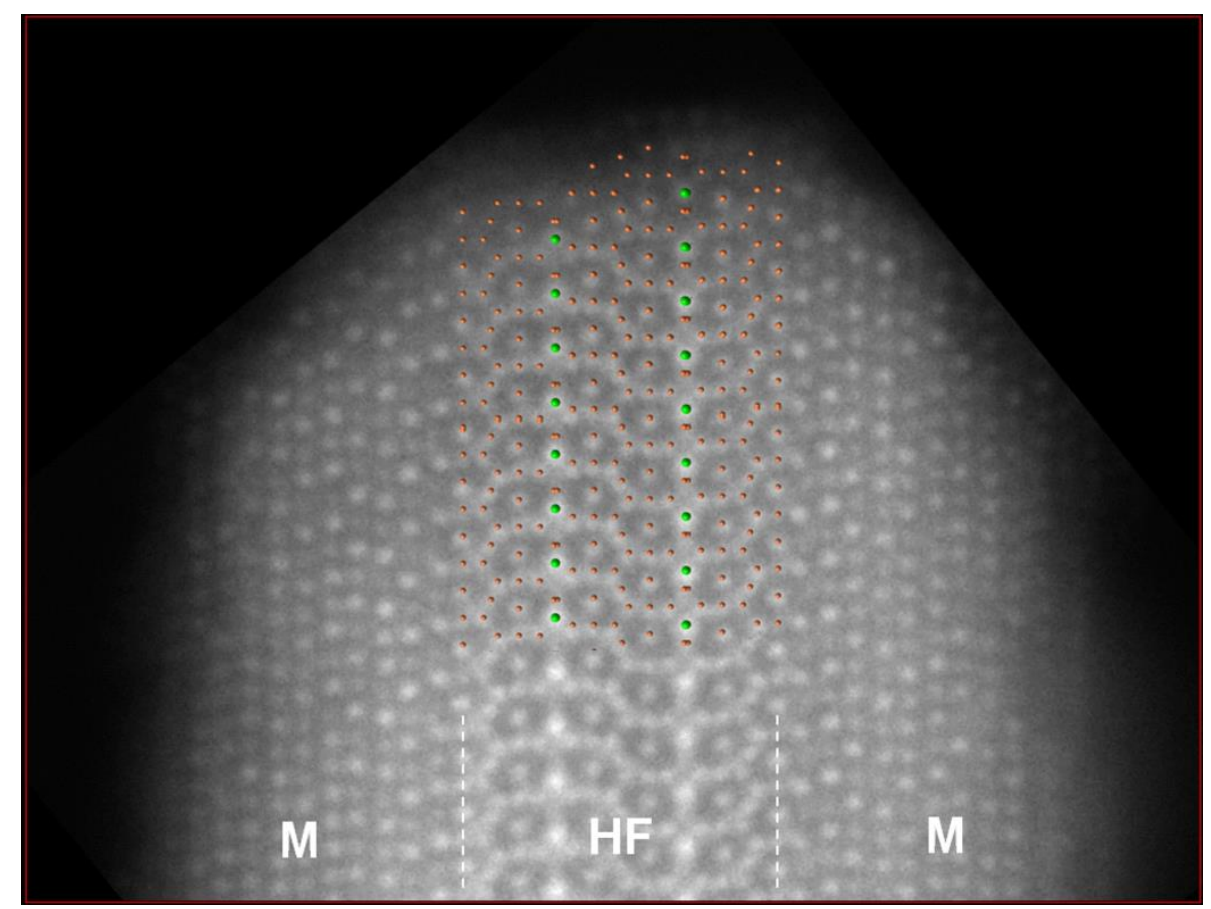

Fig. S5 HAADF STEM image of a composite nanoplatelet in the CNP2.5 sample with the superimposed hexaferrite projected structure $\left(\langle 10-10\rangle_{\mathrm{HF}} \|\langle 101\rangle_{\mathrm{M}}\right)$. 


\section{Quantitative EDXS analyses of the core nanoplatelets}

The composition of the BSHF nanoplatelets were compared with the composition of larger platelet crystals of the same $\mathrm{BaFe}_{10.5} \mathrm{Sc}_{1.5} \mathrm{O}_{19}$ nominal composition that were used as a "bulk" standard. The platelet crystals, 0.5-2 $\mu \mathrm{m}$ wide (Fig. S5) were synthesized using the same hydrothermal method as for the nanoplatelets, just with much longer time (24 hours) at the final temperature $\left(240^{\circ} \mathrm{C}\right)$. The thickness of the platelet crystals lying flat on the specimen support was estimated by computation of the zero loss electron energy loss spectroscopy (EELS) spectra to be just over $10 \mathrm{~nm}$. The spectrum taken at the large platelet crystals was used as a standard during quantization of the spectra collected at the nanoplatelets. The relative error of the $\mathrm{Fe}+\mathrm{Sc} / \mathrm{Ba}$ ratio, tested on the large platelet crystals was estimated to be less than $\pm 6 \%$.

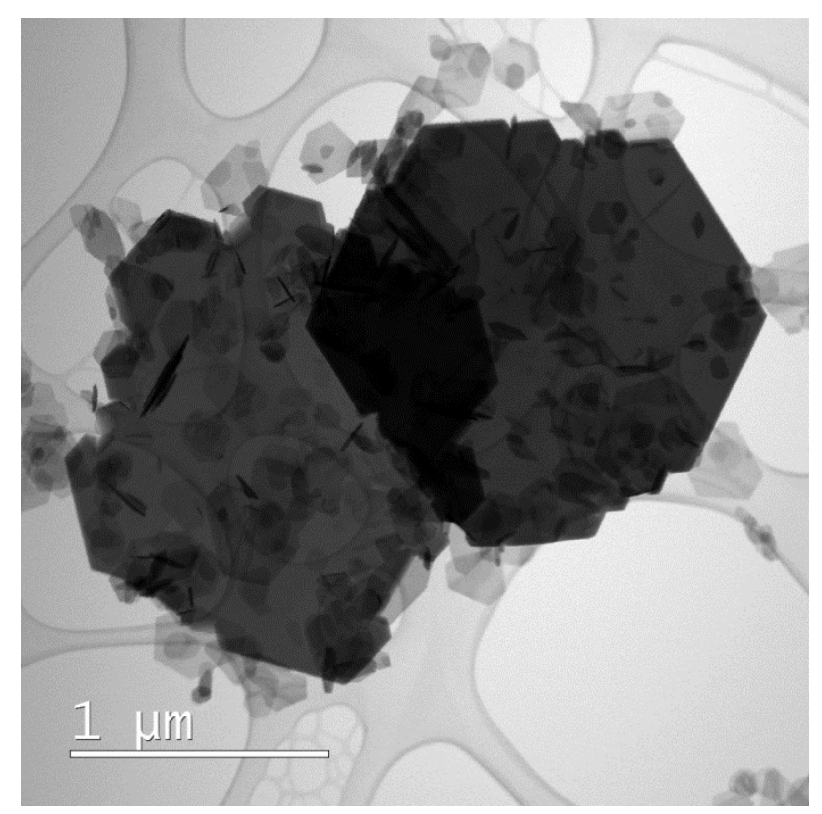

Fig. S6 BF STEM image of platelet crystals used as a "bulk" standard for EDXS analyses. 


\section{Ab-initio calculation}

To explain the reason for the experimentally-observed S-block-termination by means of the energetically most favorable structure we performed ab-initio simulations within the framework of the density-functional theory. The idea was to compare the calculated total energies $E_{t o t}$ for several S- and R-terminated structures, which all consist of a fixed number of the $\mathrm{Ba}, \mathrm{Fe}$ and $\mathrm{O}$ atoms, and to determine the most stable one with the minimum $E_{\text {tot. }}$. The calculations were carried out by applying the Quantum-Espresso $\operatorname{code}^{\mathrm{S} 7}$ with the generalized-gradient approximation (GGA) ${ }^{\mathrm{S} 8}$ of the exchangecorrelation potential. The interaction between the valence and the core electrons was described by the Troullier-Martins-type ${ }^{\mathrm{S} 9}$ pseudopotential. All considered structures were optimized by relaxing the atomic positions in terms of minimizing the total energies and inter-atomic forces. The plane-wave and the charge-density cut-off parameters were set to $204 \mathrm{eV}$ and $816 \mathrm{eV}$, respectively. The Brillouin-zone integration was carried over $4 \times 4 \times 1$ k-points evenly distributed on the Monkhorst-Pack mesh ${ }^{\mathrm{S} 10}$. The criterion for the self-consistency was the total-energy difference between the two subsequent iterations being less than $10^{-9} \mathrm{Ry}$. The structures were considered as relaxed when the magnitudes of all inter-atomic forces were less than $10^{-3}$ Ry/a.u., where a.u. stands for the Bohr radius. The thickness $d_{\text {vac }}$ of the vacuum was half of the slab thickness, in the case of the structures presented in Fig. $\mathbf{S 6} \mathrm{d}_{\mathrm{vac}}=11.6$ a.u.. 


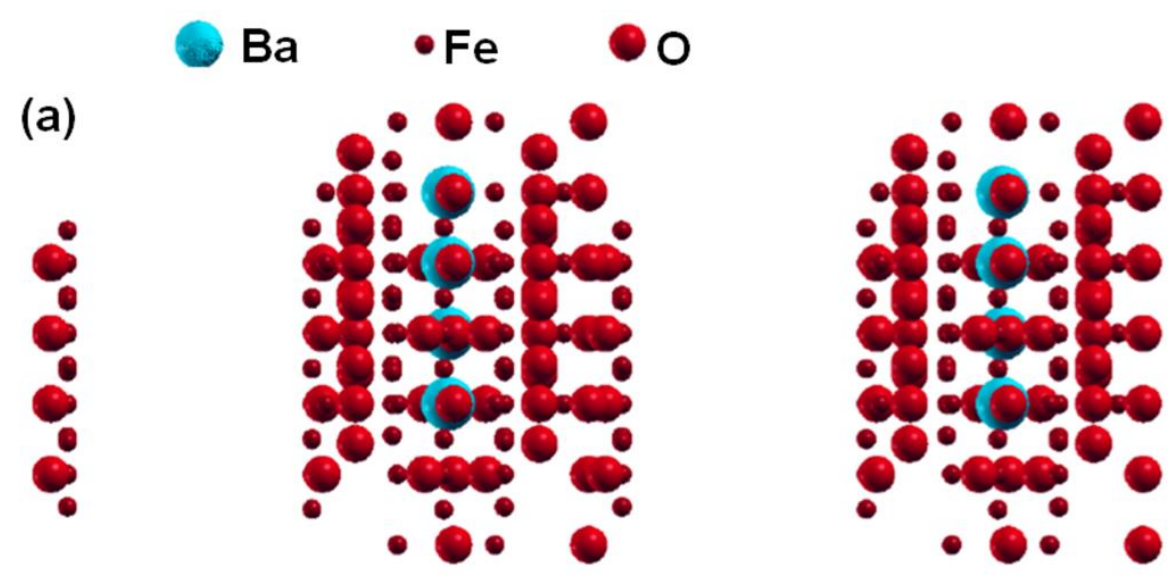

(b)
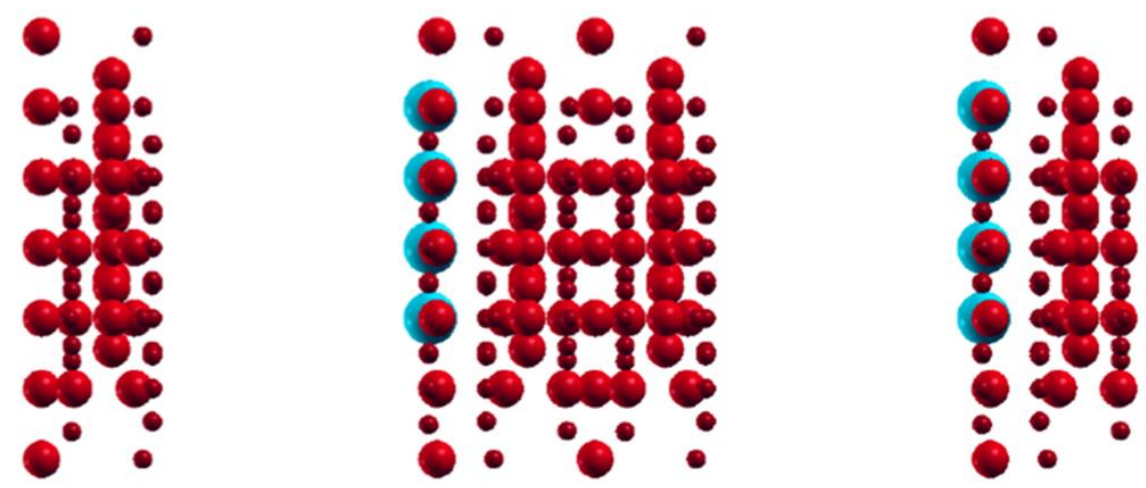

Fig. S7 An example of the modeled HF structures terminated by the Fe-only a) and Fe$\mathrm{Ba}$ b) surfaces. The calculated total energy of the (b) structure is $127 \mathrm{meV} / \mathrm{atom}$ lower than the total energy of the (a) structure. 


\section{Magnetic measurements of physical mixtures of hexaferrite nanoplatelets and maghemite nanoparticles}

To clearly show the difference between the magnetic properties of the exchangecoupled composite nanoplatelets and the hysteresis of a physical mixture of the hardmagnetic and the soft-magnetic phases, the hysteresis loop of a mixture of BSHF nanoplatelets and maghemite (M) nanoparticles was measured. As the soft phase pherical M nanoparticles, $9.7 \pm 1.5 \mathrm{~nm}$ in diameter, were chosen (Fig. S7). The volume of the M nanoparticles was comparable to that of the maghemite layers of the CNP1.9 composite nanoplatelets. First, the two types of the magnetic particles were dispersed in sucrose, as explained above. Then, the two magnetic-particles-containing sucrose powders were mixed together in a M/BSHF mass ratio of 1 , corresponding to the estimated composition of CNP1.9.

Fig. S8 shows the hystereses loops for the hard-magnetic BSHF core nanoparticles, soft-magnetic $\mathrm{M}$ nanoparticles and their physical mixture $(\mathrm{BSHF}+\mathrm{M})$. Red dashed loop represents the sum of the hysteresis loops for the two magnetic components (red loop), calculated using the formula $\boldsymbol{M}_{\text {mix }}(\boldsymbol{H})=\mathbf{0 . 5} \boldsymbol{M}_{\mathrm{BSHF}}(\boldsymbol{H})+\mathbf{0 . 5} \boldsymbol{M}_{\mathbf{M}}(\boldsymbol{H})$. Note that the mixture $\mathrm{BSHF}+\mathrm{M}$ shows the characteristic constricted hysteresis loop for two phases that magnetize independently.
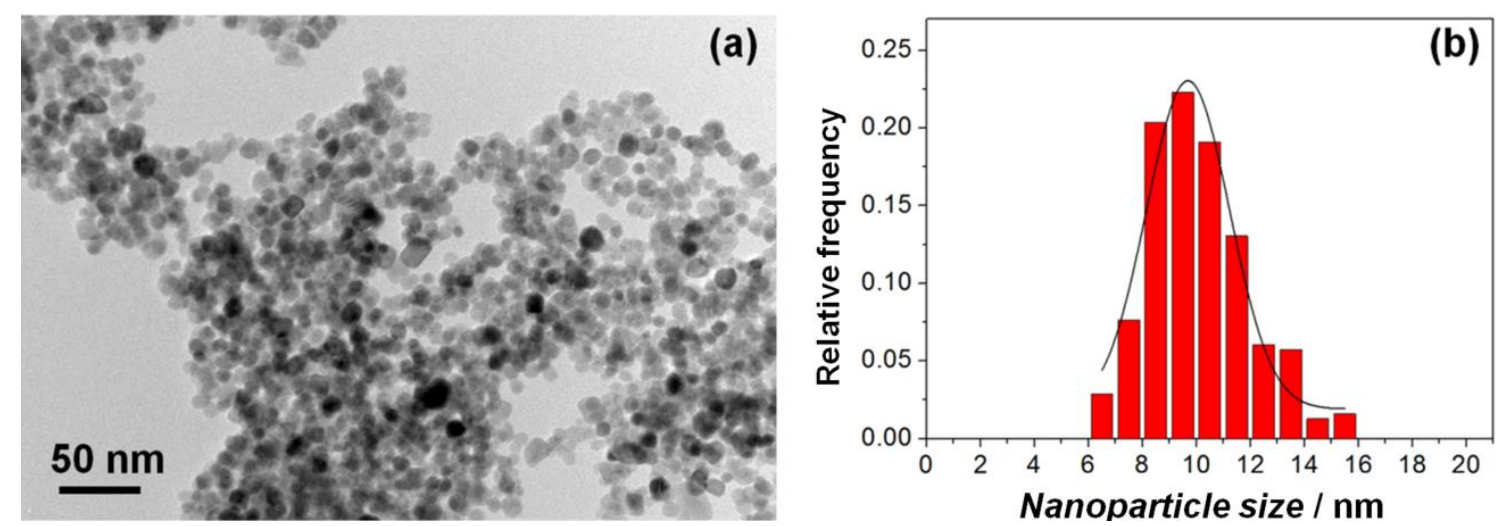

Fig. S8 TEM image (a) and particle size distribution (b) of maghemite M nanoparticles used as soft-magnetic phase for magnetic measurements of the physical mixtures. 


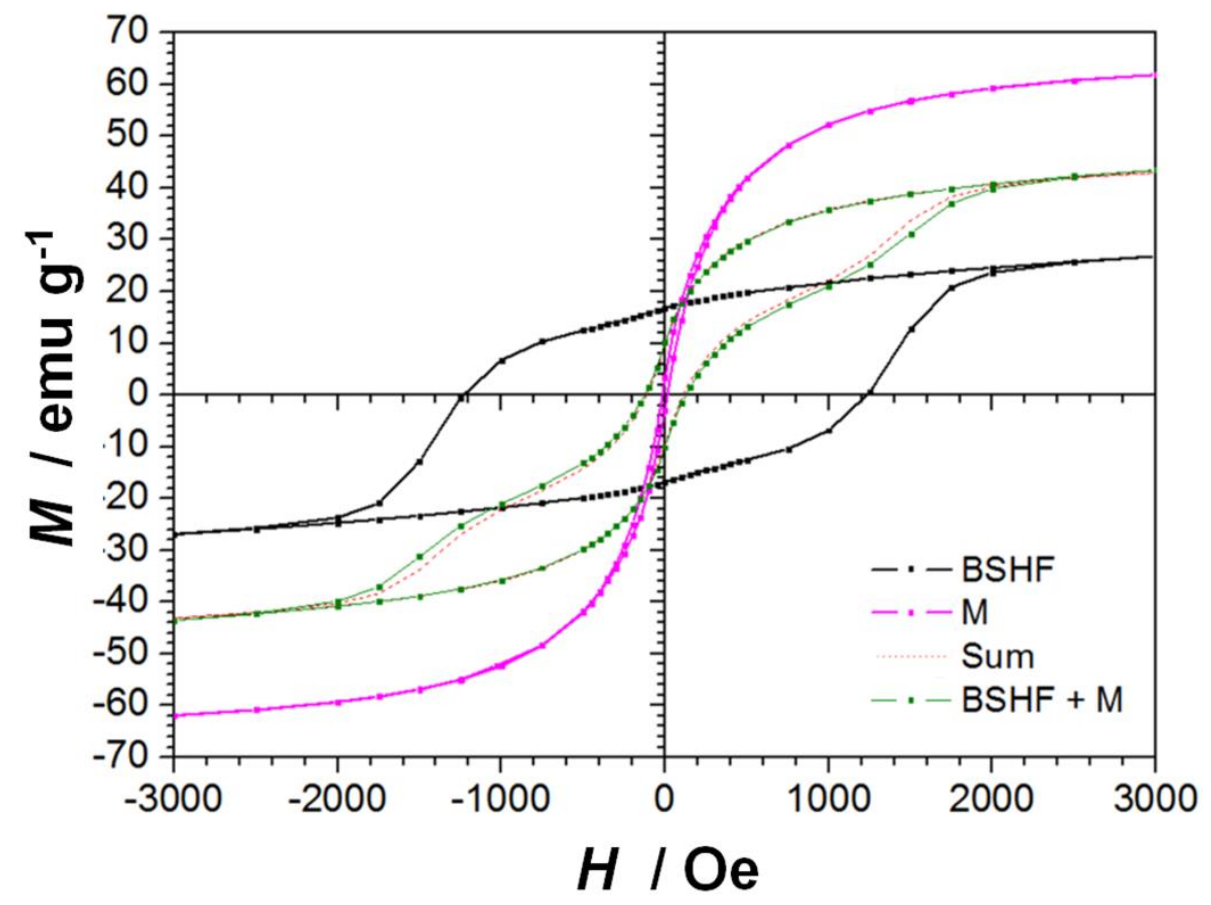

Fig. S9 Magnetic hystereses for the BSHF nanoplatelets, the M nanoparticles and their physical mixture. 


\section{Properties of the composite nanoplatelets composed of hexaferrite core coated with non-magnetic zinc-ferrite layers}

To assess the influence of the epitaxial layers on the magnetic properties of the BSHF nanoplatelet core, non-magnetic zinc-ferrite layers were deposited instead of $\gamma-\mathrm{Fe}_{2} \mathrm{O}_{3}$. The zinc ferrite was deposited using the same procedure as used for synthesis of the CNP1.9, except that $\mathrm{FeCl}_{2}$ was replaced by $\mathrm{ZnCl}_{2}$. The TEM analysis (Fig. S9) showed only composite nanoplatelets (without any uncoupled ferrite particles), while EDXS analysis in the STEM confirmed that each nanoplatelet contained $\mathrm{Zn}$. Elemental mapping recorded by EDXS in STEM (Fig. S10) clearly shows presence of $\mathrm{Zn}$ in the layers. Note that similar to the of $\gamma-\mathrm{Fe}_{2} \mathrm{O}_{3}$ layers, the $\mathrm{Zn}$-ferrite also grows epitaxially on the BSHF platelets (Fig. S10).

In bulk, zinc ferrite $\left(\mathrm{ZnFe}_{2} \mathrm{O}_{4}\right)$ is paramagnetic at room temperature, as the tetrahedral $\mathrm{A}$ sublattice of its normal spinel structure is almost exclusively populated by the nonmagnetic $\mathrm{Zn}^{2+}$ ions. ${ }^{\mathrm{S} 11, \mathrm{~S} 12}$ However, in nanostructured form, zinc ferrite becomes weakly ferrimagnetic, as a significant proportion of the $\mathrm{Fe}^{3+}$ ions exchanges sites with the $\mathrm{Zn}^{2+}$ ions, i.e., it adopts a partially inverted spinel structure. ${ }^{\mathrm{S} 13}$ Spherical zinc-ferrite nanoparticles of approximately $8 \mathrm{~nm}$ display superparamagnetism with $\boldsymbol{M S}_{\boldsymbol{S}}$ of approximately $8 \mathrm{emu} / \mathrm{g}$ (measured at $13.8 \mathrm{kOe}$ ). ${ }^{[\mathrm{S} 13]}$ With annealing at temperatures above approximately $400{ }^{\circ} \mathrm{C}$ in air, their structure rearrange to the equilibrium "bulk" state and the nanoparticles became paramagnetic. ${ }^{\mathrm{S} 13}$ The measurement of the $\mathrm{Zn}$-ferrite based composite nanoplatelets, ZnCNP, with approximately $1.9 \mathrm{~nm}$ thick zinc-ferrite layers showed a single-phase hysteresis loop (Fig. S11). The $\boldsymbol{M}_{\boldsymbol{S}}$ of the as-synthesized ZnCNP was lower to that of the BSHF nanoplatelets indicating weakly magnetic layers. Estimation of the $\boldsymbol{M S}_{\boldsymbol{S}}$ of zinc ferrite considering its mass fraction in the composite (wlayer $\square$ 0.5) gave $M_{S(l a y e r)}$ of approximately $13 \mathrm{emu} / \mathrm{g}$. However, annealing the ZnCNP at 500 ${ }^{\circ} \mathrm{C}$ the $\boldsymbol{M S}_{S}$ of the $\mathrm{ZnCNP}$ decreased almost to the half of the one of the BSHF nanoplatelets, indicating that the zinc ferrite became nearly paramagnetic. Moreover, the zinc-ferrite layers had pronounced influence on the $\boldsymbol{H}_{\boldsymbol{C}}$ of the composite nanoplatelets, which exhibit considerably smaller $\boldsymbol{H}_{\boldsymbol{C}}$ than the BSHF core. Given the non-magnetic character of zinc-ferrite, the most plausible origin for the $\boldsymbol{H}_{\boldsymbol{C}}$ decrease is the structural stresses induced on the core by the epitaxial surface layers. 

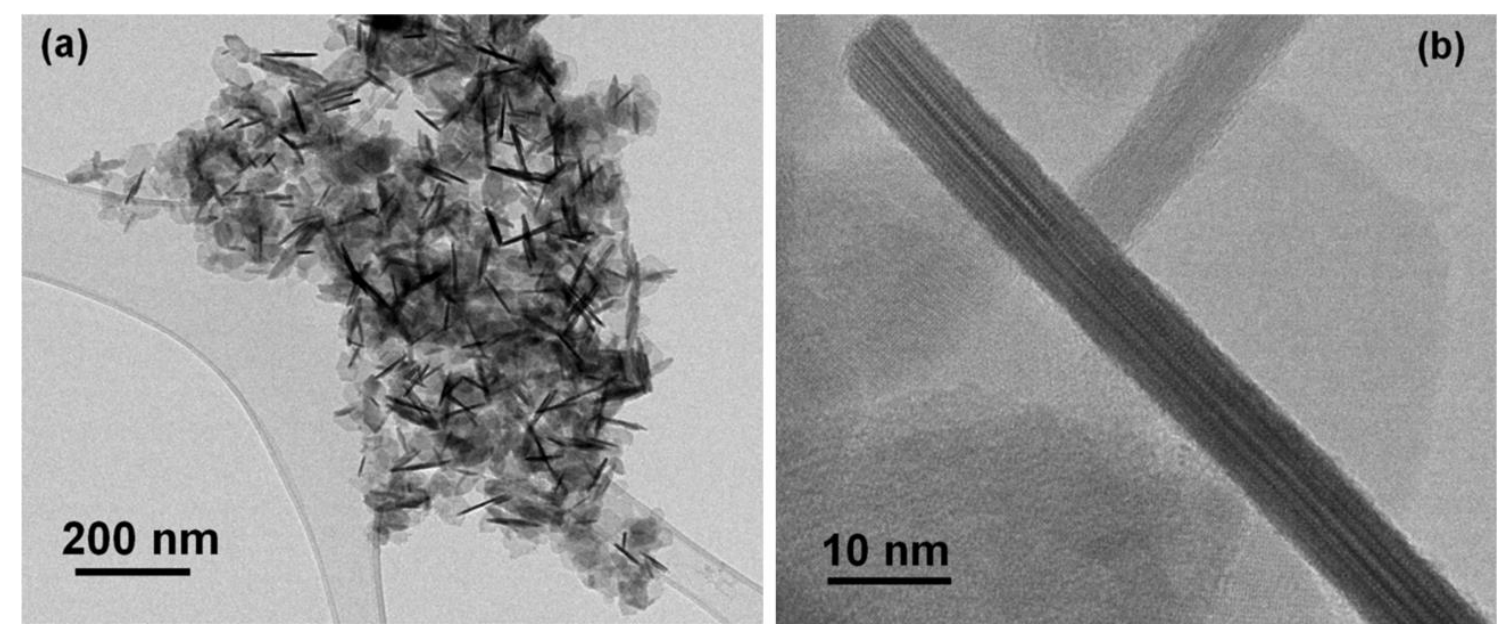

Fig. S10 TEM (a) and HRTEM (b) images of the ZnCNP nanoplatelets.
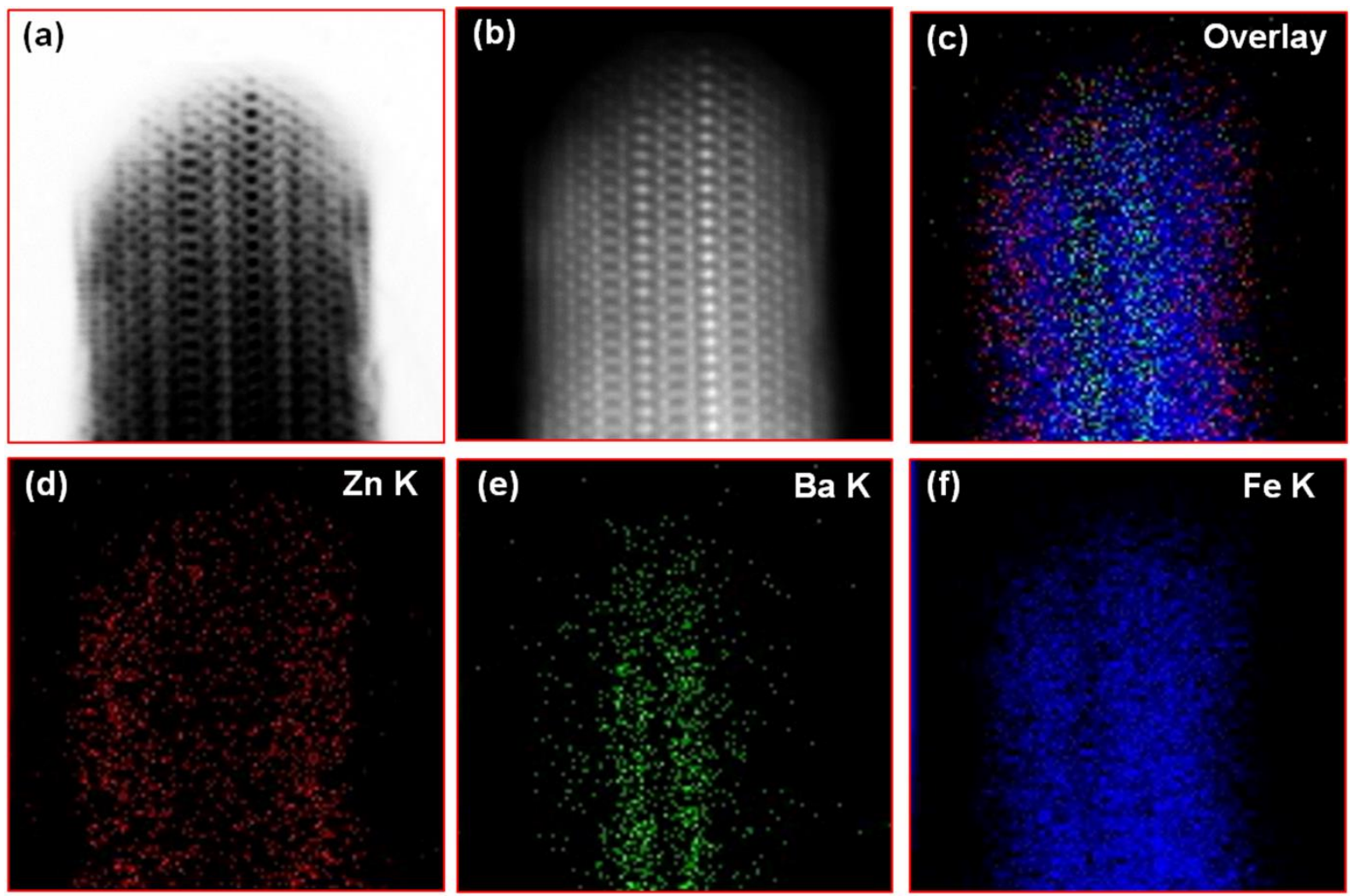

Fig. S11 BF (a), HAADF (b) STEM images and the corresponding EDXS elemental mappings (c-f) of the composite ZnCNP nanoplatelet oriented with its hexaferrite (HF) core along $\langle 11-20\rangle_{\mathrm{HF}}$. 


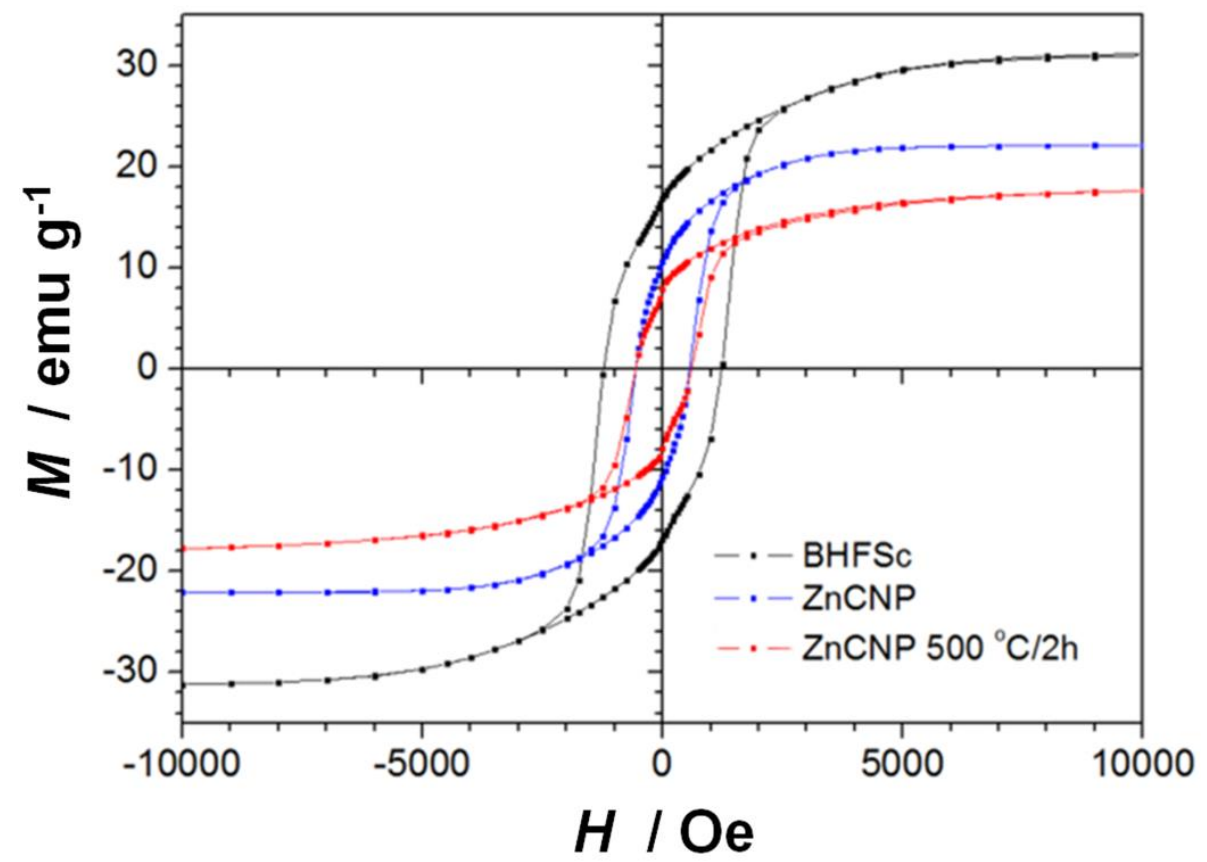

Fig. S12 Hystereses loops for the BSHF core nanoplatelets and the composite nanoplatelets $\mathrm{ZnCNP}$, before and after annealing. 


\section{High-temperature magnetic measurements}

To minimize growth and excessive agglomeration of the nanoplatelets during the hightemperature magnetic measurements they were coated with thin layer of silica, $\mathrm{SiO}_{\mathrm{x}}$, The BSHF core nanoplatelets were coated with $\sim 8 \mathrm{~nm}$ thick silica layer (Fig. S12), whereas the coating was thinner at the CNP1.1 nanoplatelets, approximately $3 \mathrm{~nm}$ (Fig. S13).

Room temperature hysteresis loops were measured for both samples (Fig. S14) before and after the $M(\mathrm{~T})$ measurements up to $550{ }^{\circ} \mathrm{C}$ (Fig. 4c). It can be seen that while the magnetic properties of the BSHF core nanoplatelets only change slightly (perhaps due to some strain relieve after reaching $550{ }^{\circ} \mathrm{C}$ ), the ones for the CNP1.1 nanoplatelets are markedly different before and after the $\mathrm{M}(\mathrm{T})$ measurements. The large change in $\boldsymbol{M S}_{\boldsymbol{S}}$ and $\boldsymbol{H}_{\boldsymbol{C}}$ hint a possible structural transition CNP1.1 nanoplatelets after the high temperature treatment.

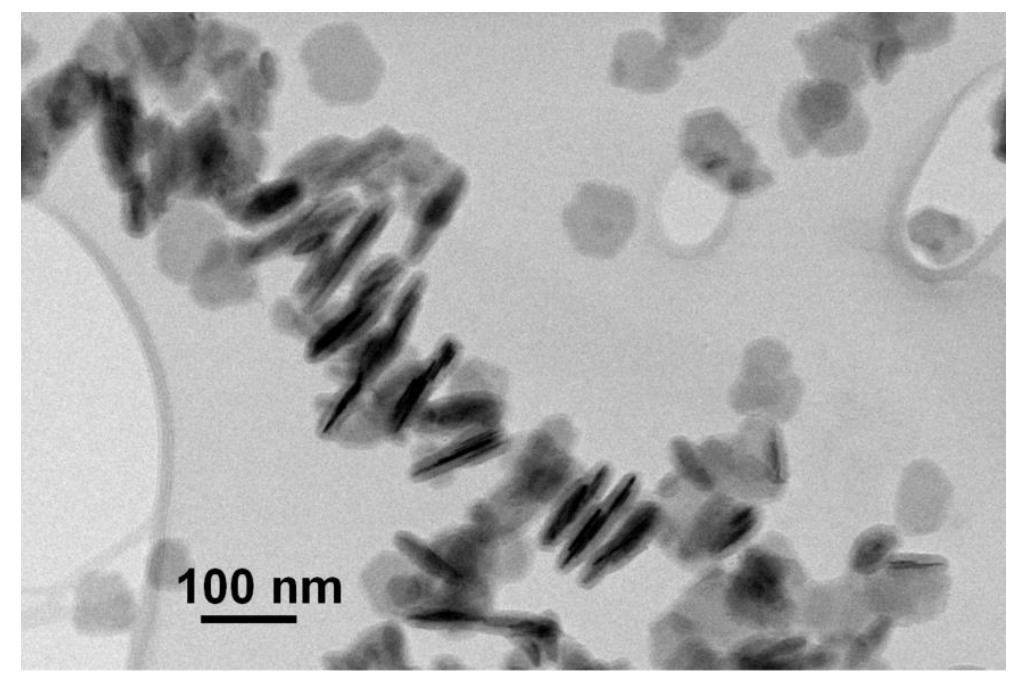

Fig. S13 TEM image of the silica-coated BSHF nanoplatelets 


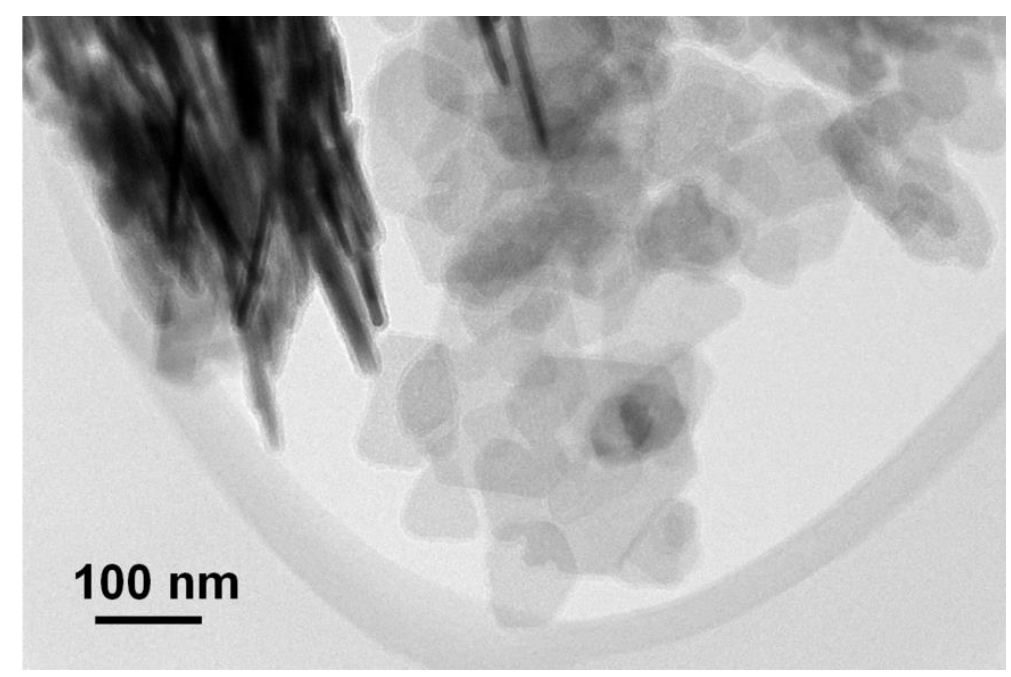

Fig. S14 TEM image of the silica-coated CNP1.1 nanoplatelets.
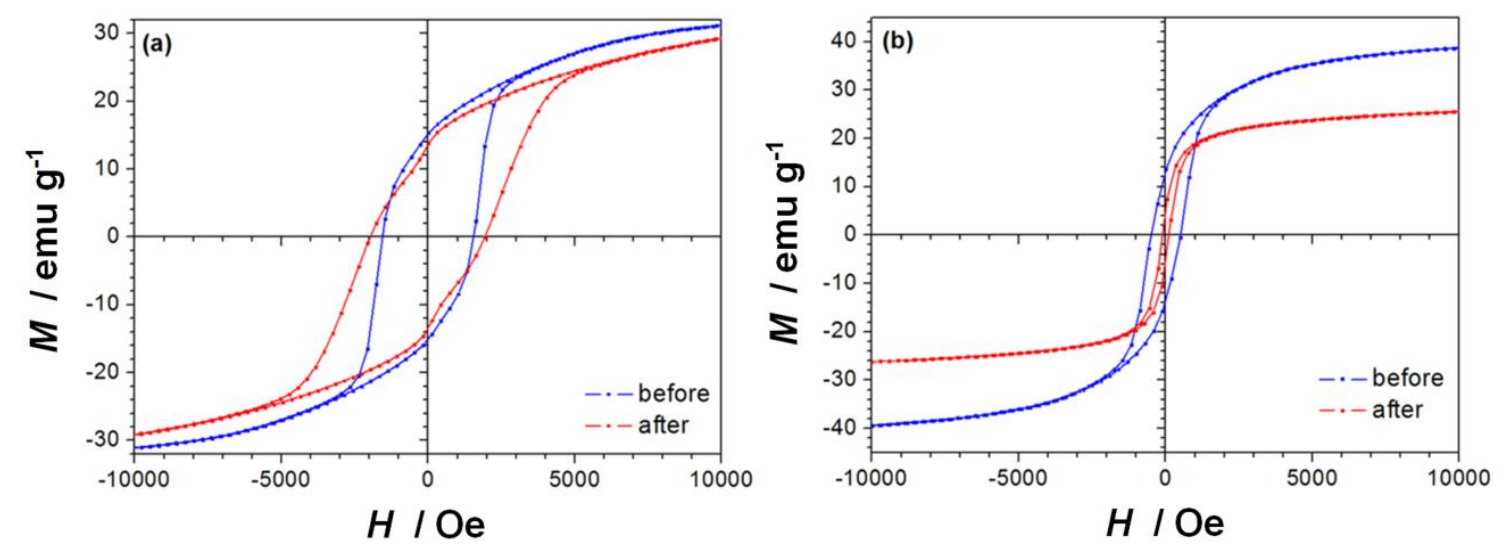

Fig. S15 Room-temperature magnetic hysteresis loops for the silica coated BSHF (a) and CNP1.1 (b) nanoplatelets measured before and after the high temperature measurements. 


\section{References}

S1. S. Asuha, S. Zhao, X. H. Jin, M. M. Hai and H. P., Bao, Appl. Surf. Sci., 2009, 255, 8897-8901.

S2. K. Koch, Determination of Core Structure Periodicity and Point Defect Density along Dislocations, Arizona State University, 2002.

S3. S. Kralj, D. Makovec, S. Čampelj and M. Drofenik, J. Magn. Magn. Mater., 2010, 322, 1847-1853.

S4. J. H. Monkhorst and J. D. Pack, Phys. Rev. B., 1976, 13, 5188-5192.

S5. R. M. Cornell, U. Schwertmann, The Iron Oxides: Structure, Properties, Reactions, Occurrences and Uses, Wiley-VCH, Weinheim, Germany, 2003.

S6. I. Chamritski, G. Burns, J. Phys. Chem. B, 2005, 109, 4965-4968.

S7. Giannozzi, P., Baroni, S.,, Bonini, N., Calandra, M:, Car, R., Cavazzoni, C., D. Ceresoli, G.L. Chiarotti, M. Cococcioni, I. Dabo, A.D. Corso, S. de Gironcoli, S. Fabris, G. Fratesi, R. Gebaurer, U. Gerstmann, C. Gougoussis, A. Kokalj, M. Lazzeri, L. Martin-Samos, N. Marzari, F. Mauri, R. Mazzarello, S. Paolini, A, Pasquarello, L. Paulatto, C. Sbraccia, S. Scandolo, G. Sclauzero, A.P. Seitsonena, A. Smogunov, P. Umari and R.M. Wentzcovitch, J. Phys. Condens. Matte, 2009, 21, 395502.

S8. J. P.Perdew, K. Burke and M. Ernzerhof, M. Phys. Rew. Lett., 1996, 77, 38653868 .

S9. N. Troullier and J.L. Martins, Phys. Rev. Lett., 1991, 43, 1993-2006.

S10. J. H. Monkhorst, J. D. Pack, Phys. Rev. B., 1976, 13, 5188-5192.

S11. H. S. O’Neill, Eur. J. Mineral., 1992, 4, 571-580.

S12. D. Makovec, A. Kodre, I. Arčon, J. Nanopart. Res., 2011, 13, 1781-1790.

S13. D. Makovec and M. Drofenik, J. Nanopart. Res., 2008, 10, 131-141. 